Article

\title{
A Dynamic Approach for Faster Performance Measurements on Hydraulic Turbomachinery Model Testing
}

\author{
Vlad Hasmatuchi ${ }^{1, *(1)}$, Alin Ilie Bosioc ${ }^{2}$, Sébastien Luisier ${ }^{1}$ and Cécile Münch-Alligné ${ }^{1}$ (D) \\ 1 School of Engineering, Systems Engineering Institute, University of Applied Sciences and Arts Western \\ Switzerland Valais, Route du Rawyl 47, CH-1950 Sion, Switzerland; sebastien_luisier@hotmail.com (S.L.); \\ cecile.muench@hevs.ch (C.M.-A.) \\ 2 Department of Hydraulic Machinery, University Politehnica Timisoara, Bv. Mihai Viteazu 1, \\ RO-300222 Timisoara, Romania; alin.bosioc@upt.ro \\ * Correspondence: vlad.hasmatuchi@hevs.ch; Tel.: +41-27-606-8825
}

Received: 20 July 2018; Accepted: 14 August 2018; Published: 21 August 2018

check for updates

Featured Application: Reducing the time required to assess or improve the performances of hydraulic machines is a crucial aspect for small-hydro applications. Indeed, the investments for applied research and development for this type of technologies are limited. In this paper, an alternative approach to measure the performance is evaluated and came out to be up to ten times faster than standard methods.

\begin{abstract}
During the design and optimization of hydraulic turbomachines, the experimental evaluation of hydraulic performances beyond the best efficiency point and for off-design conditions remains essential to validate the simulation process and to finalize the development. In this context, an alternative faster method to measure the efficiency of hydraulic turbomachines using a dynamic approach has been investigated. The so-called "sliding-gate" dynamic measurement method has been adapted and implemented on the hydraulic test rig of the HES-SO Valais/ / Wallis, Sion, Switzerland. This alternative approach, particularly gainful for small-hydro for which the investment devoted to development is limited, has been successfully assessed on two cases for drinking water networks energy recovery. A $2.65 \mathrm{~kW}$ double-regulated laboratory prototype of a tubular axial micro-turbine with two independent variable speed counter-rotating runners and a $11 \mathrm{~kW}$ multi-stage centrifugal pump-as-turbine (PAT) with variable speed have been selected. The hydraulic efficiency results obtained by dynamic measurements are compared to the ones obtained by the classical steady point-by-point method. This dynamic method, suitable not only for hydraulic machinery, allows: (i) reducing significantly (up to $10 \times$ ) the time necessary to draw the complete efficiency characteristics of a hydraulic machine; (ii) rapidly detecting the hydrodynamic instabilities within the operating range of the machine.
\end{abstract}

Keywords: dynamic measurements; experimental testing; efficiency; hydraulic turbomachines; small-hydro

\section{Background}

Hydropower, small and large, remains the most important source of renewable energy for electrical power production providing $16.4 \%$ of the world electricity mix in 2016 [1]. In Switzerland, hydropower provides $56.7 \%$ of the electricity of the country, $5.7 \%$ coming from small-hydro (see [2]), considering plants with an "annual average used" capacity up to $10 \mathrm{MW}$ [3]. Indeed, there are more than 1300 small-scale hydropower plants in operation, with an installed capacity of approximately 
$760 \mathrm{MW}$ and an output of $3400 \mathrm{GWh}$ per year. In the post-Fukushima era, Switzerland has decided to renounce to its nuclear energy power stations and to accelerate the transition to a sustainable energy future based on carbon-free renewable electricity sources. To increase the small hydropower production while limiting the environmental impact, the primary sustainable solution would be to harness the energy of existing infrastructure, such as drinking water networks or wastewater networks.

To address such potential sites, axial flow turbines provide low CAPEX solution by limiting the civil engineering works. Along the years, different research teams developed various models of micro hydro turbines. Ruprecht et al. $[4,5]$ studied numerically and experimentally a new axial turbine designed for pressure recuperation on piping systems. The turbine consists of a stator with 12 blades and a rotor with 15 blades, with the possibility to modify the angle of the guide vanes. Alexander et al. [6] designed and tested four different axial microturbines for heads between $4 \mathrm{mWC}$ and $9 \mathrm{mWC}$ with a hydraulic efficiency around $68 \%$. Samora et al. [7] investigated experimentally the behavior of a five-blade axial turbine reaching $64 \%$ of efficiency for a head of $3.4 \mathrm{mWC}$. In order to achieve a higher efficiency and a stable operation of this type of microturbines, axial turbines with counter rotating runners have been developed. Accordingly, Sonohata et al. [8] studied numerically such turbines with a calculated efficiency for both runners of $70 \%$, with a drastically decreased efficiency for the rear runner at partial and large flow rates.

In this framework, a research project to develop new technologies to harvest hydraulic energy on existing infrastructure has been set up by the HES-SO Valais/ / Wallis (Sion, Switzerland) and the EPFL-Laboratory for Hydraulic Machines, Lausanne, Switzerland. One of the results is a new axial microturbine with counter-rotating runners for drinking water networks [9-11], which should cover a part of the hydraulic energy potential that must be additionally exploited until 2050 in Switzerland. The microturbine with a shroud diameter of $\phi 0.1 \mathrm{~m}$ and a hub diameter of $\phi 0.08 \mathrm{~m}$ was designed to reach a hydraulic efficiency of more than $85 \%$. The nominal conditions are expected for a discharge of $8.71 \cdot \mathrm{s}^{-1}$, a head of $15 \mathrm{mWC}$ on each runner and a rotational speed of $3000 \mathrm{~min}^{-1}$ for both runners. The prototype turbine was manufactured and tested on the HES-SO Valais//Wallis-Switzerland test rig [12] to assess its performances.

According to Münch-Alligné and Avellan [13], the budget allocated to the development for small-hydro is much more limited compared to large-hydro. The total men-hours investment dedicated to the performance measurements must be reduced as much as possible. To this end, with the main purpose of reducing the time necessary to perform the full hydraulic performance tests of a turbomachine, an alternative dynamic method has been implemented and validated. Based on dynamic measurements to retrieve the hydraulic characteristics, the so-called sliding-gate method was first tested by Almquist et al. [14,15]. This method relies on same measurements and instrumentations than classical efficiency method, the only difference exists in continuous modification of one parameter: the angle of the guide vanes or the speed of the runner. It presents the advantage of obtaining continuous efficiency curves over the entire operating range while reducing significantly the total time required performing the tests $[16,17]$. This approach could be also used for fast detection of the different operating regions where hydraulic instabilities arise in the machine, such as part-load and full-load cavitating vortex ropes, harmful rotor-stator pressure fluctuations and vibrations, or even flow rotating-stalls [18]. Indeed, Hasmatuchi [19] shown that a rotating-stall developing in a reduced scale model of a pump-turbine can be successfully detected by pressure fluctuation measurements during a transient test over the S-region of its characteristic in turbine mode.

The alternative dynamic method tested in this study allows us to:

(i) reduce significantly the time necessary to draw the complete efficiency characteristics of a hydraulic machine;

(ii) detect hydrodynamic instabilities within the operating range of the machine;

(iii) establish an alternative/complementary faster procedure for hydraulic efficiency measurements. 
The current work comes with two examples of implementation and validation of dynamic efficiency measurements on hydraulic turbomachinery model testing: a double-regulated axial counter-rotating variable speed microturbine and a centrifugal multi-stage variable speed pump-as-turbine (PAT). The experimental methodology to obtain the hydraulic efficiency hill-chart from measurements using the static and the dynamic methods is presented. Then, the experimental setup, including the employed hydraulic test rig and the two case studies are introduced. The instrumentation and the testing protocol are also provided. The results focus first on the selection and validation of the optimal acceleration/deceleration ramps of the runners to ensure a steady-state condition for the dynamic measurements. Finally, the resulting hydraulic hill-charts obtained by the two methods are compared. A discussion and concluding remarks come to complete the work.

\section{Methodology}

The main parameters necessary to retrieve the hydraulic performance of a turbomachine are the specific energy $\mathrm{E}$, the discharge $\mathrm{Q}$, the rotational speed of the runner $\mathrm{N}$, the mechanical torque $\mathrm{T}_{\mathrm{m}}$ and the pressure at the low side of the machine. With these measured parameters, the E-Q operating range along with the corresponding hydraulic efficiency and the cavitation behavior can be evaluated. In addition, measurements of the runaway characteristic, different loading forces, pressure fluctuations and observations on the draft tube vortex rope development come to complete a typical set of tests [20]. Finally, the dimensionless characteristics (e.g., using the discharge-energy coefficients $\phi-\psi$, or the speed-discharge factors $\mathrm{N}_{\mathrm{ED}}-\mathrm{Q}_{\mathrm{ED}}$, etc.) retrieved on the reduced-scale model present the advantage of being valid for the target prototype as well, respecting both the geometric and hydraulic similitude laws.

\subsection{Classical Standard Static Measurements Approach}

Considering the IEC 60193 [21] standard recommendations, the performance test shall be performed at constant speed or at constant testing head. To facilitate the results post-processing, it is recommended to perform the tests at constant speed and water temperature in order to maintain the Reynolds number approximately constant. Then, to draw the tri-dimensional hydraulic efficiency surface, a sufficient number of measurement points covering the entire operating range must be considered.

Now let us focus on a double-regulated turbine, such as a micro-turbine with two independent counter-rotating variable speed runners. In terms of hill-chart construction approach, the case is similar to a Kaplan turbine with variable angles of guide vanes and runner blades. Once the set of measurement points selected, the classical standard measurement approach consists of regulating one operating point, waiting several tens of seconds to ensure a stabilized flow condition and then perform the measurement, recording usually the average and the standard deviation values of each parameter from signals of several seconds of acquisition.

The three main sequences of measurement/processing operations to draw the final efficiency hill-chart of the machine are illustrated in Figure 1. The first step consists on several sets of measurements at constant testing head $\mathrm{H}_{\mathrm{i}}$, using combinations of turbine runners' speeds $\left(\mathrm{N}_{\mathrm{A}}\right.$ and $\mathrm{N}_{\mathrm{B}}$ ) while keeping constant the speed ratio between the two runners, $\alpha_{\mathrm{i}}$. For each selected testing head, the result is a tri-dimensional efficiency hill-chart, figured in the right side of the first step. Then, in the second step, for each testing head, the variations of efficiency $\eta$ with the discharge $Q$ and with the head $\mathrm{H}$ are drawn for all sets of constant runners' speed ratio, and their envelope is calculated. In the third phase, the previously obtained envelopes are used to construct the final full 3D hill-chart of the machine by surface interpolation. 


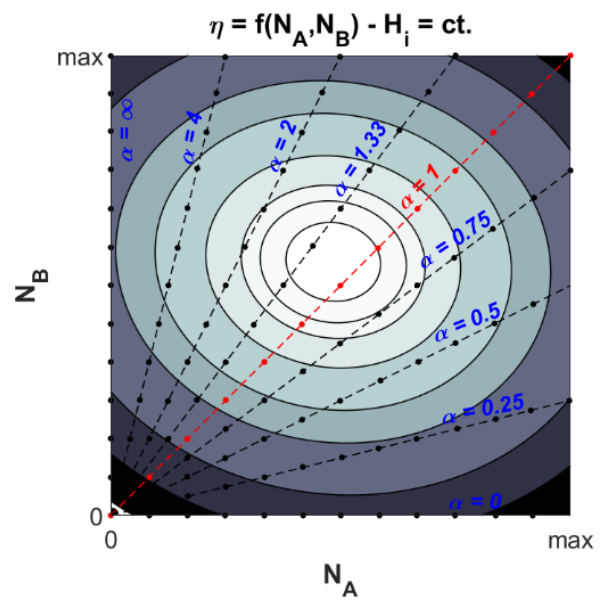

(1)

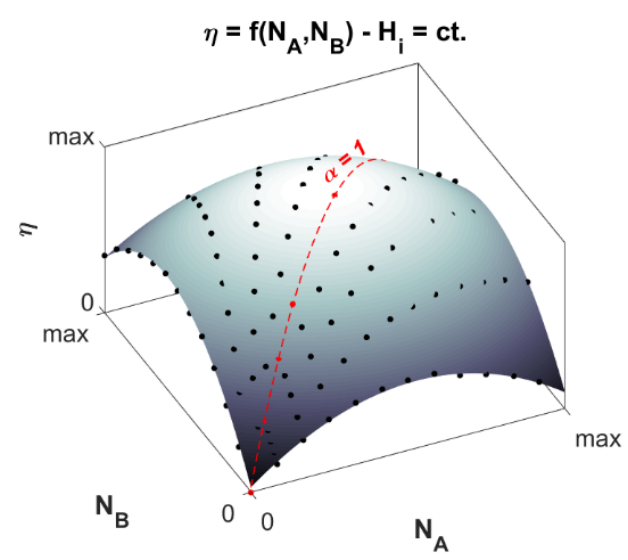

(a)

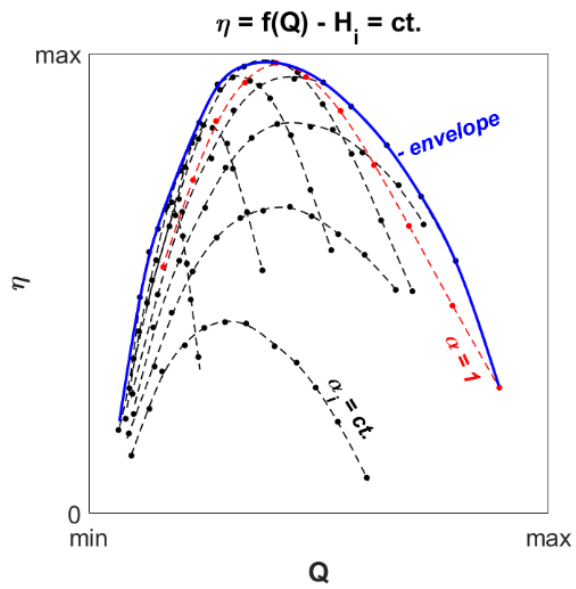

(2)

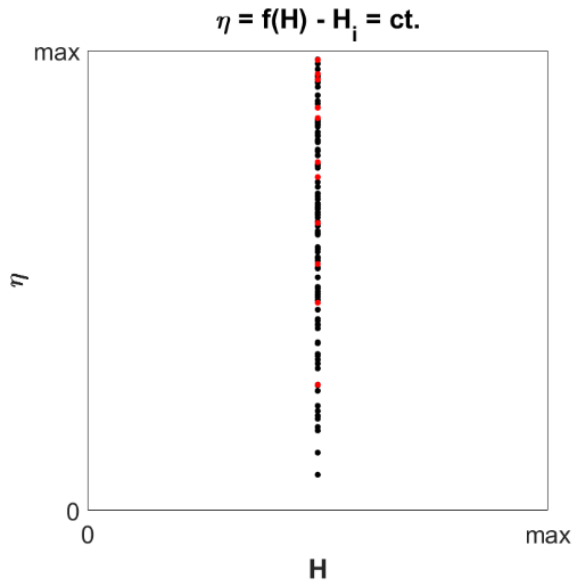

(b)

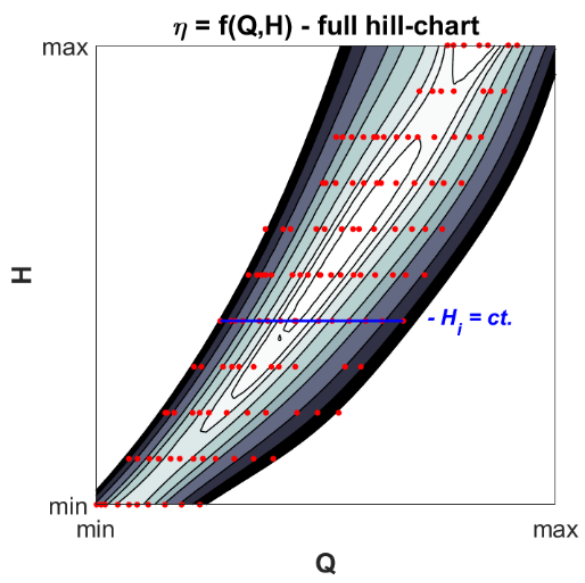

(3)

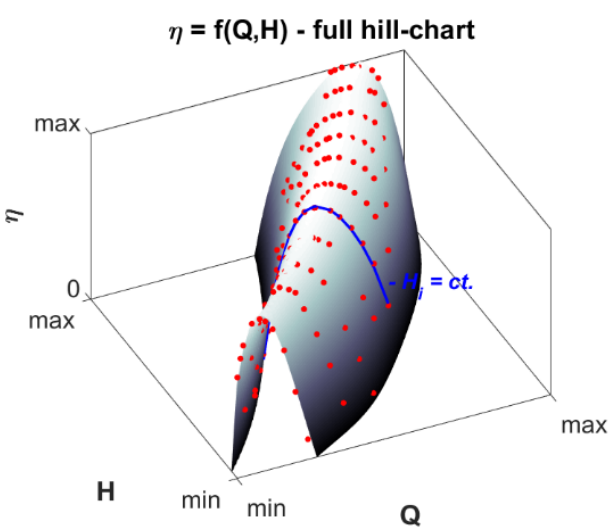

(c)

Figure 1. Standard methodology to reconstruct the full hydraulic efficiency hill-chart of a double-regulated machine using the classical static measurements method: (a) First step: measurements of several efficiency hill-charts at constant testing head $\mathrm{H}_{\mathrm{i}}$ and constant speed ratio between the two runners, $\alpha_{i}$; (b) Second step: calculation of discharge-efficiency and head-efficiency envelopes for all testing heads; (c) Third step: construction of the final full 3D hill-chart of the machine by surface interpolation using the previously obtained envelopes. 


\subsection{Alternative Dynamic Measurements Approach}

The main advantage of the classical standard point-by-point efficiency measurements approach on model testing is that it is largely proved and it allows for high-precision measurements. However, to determine the complete hill chart diagram of a turbine, a couple of days or weeks are usually necessary. From the point of view of small-hydro development, for which the investment is much more limited compared to large hydro, the classical method remains laborious and time expensive. An alternative faster solution is derived from the "sliding gate" dynamic method (Almquist et al. [15]), successfully used for index testing of Francis and Kaplan units. However, quasi steady-state flow conditions must be absolutely ensured during measurements in order to avoid errors mainly due to slow response capabilities of standard sensors and operation hysteresis.

Considering again the double-regulated turbine installed on a classical hydraulic test rig, the normal way to perform the dynamic measurements would be to vary dynamically the rotational speed of the runners while keeping constant the head. This is unfortunately almost impossible to do with the standard equipment of the test rig, even with a very reactive PID regulator that controls then speed of the recirculating pumps. In the case of prototype index tests, the head is mainly the result of the dams' levels, which will not vary during one set of tests. In laboratory model testing the measurements are therefore performed at fixed recirculating pumps' rotational speed instead of constant head.

The three main sequences of measurement/processing operations to draw the final efficiency hill-chart of the machine using the dynamic method are illustrated in Figure 2. During the first step, for each selected constant rotational speed $\mathrm{N}_{\mathrm{p} 1,2,3 \mathrm{i}}$ of the test rig recirculating pumps, the turbine runners speed is changed from zero to maximum and back to zero, while maintaining a constant speed ratio. The resulting efficiency hill-chart displayed in the right side is obtained this time not from discrete points, but from curves. Moreover, the amount of information acquired in a relatively short time is much more important compared to the few points measured by the static point-by-point method. Then, in the second phase, for each recirculating pumps' speed, the variations of efficiency $\eta$ with the discharge $\mathrm{Q}$ and with the head $\mathrm{H}$ are drawn for all sets of constant turbine runners speed ratio, and their envelopes are calculated. One may state here the completely different variation of the efficiency with the head compared to the classical static method due to the intersection of recirculating pumps' characteristic with the characteristic of the turbine. Finally, in the third phase, the obtained envelopes are again used to construct the final full 3D hill-chart of the machine by surface interpolation.
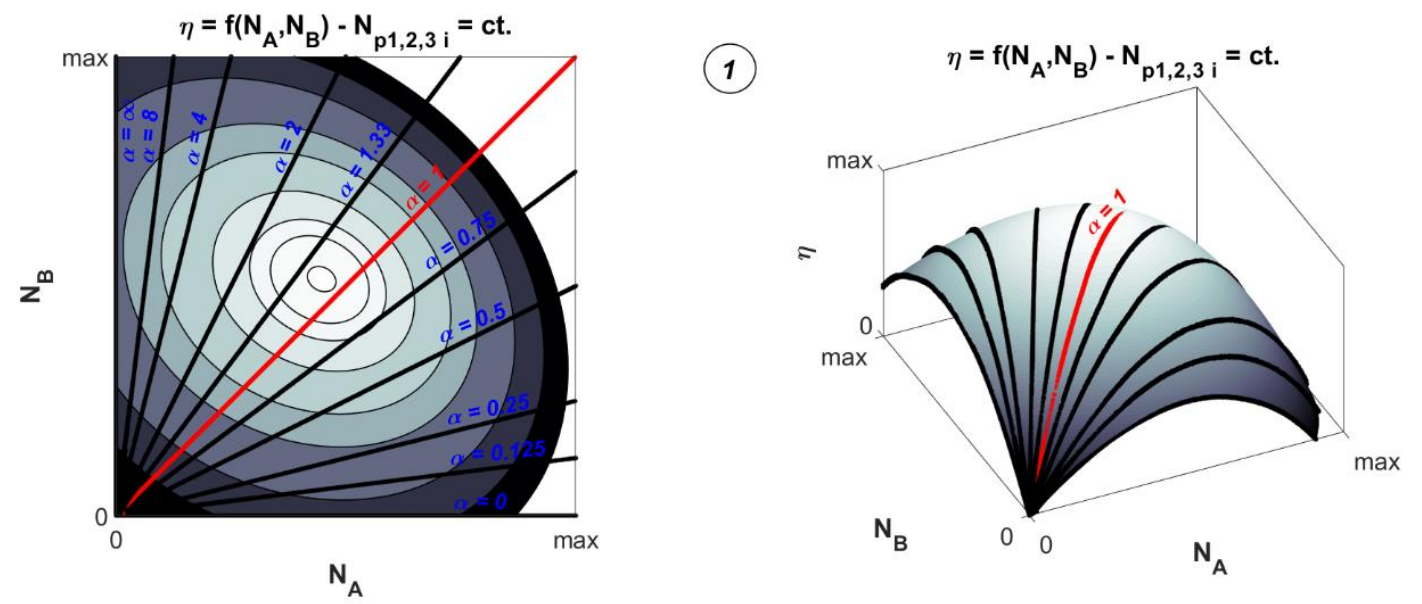

(a)

Figure 2. Cont. 

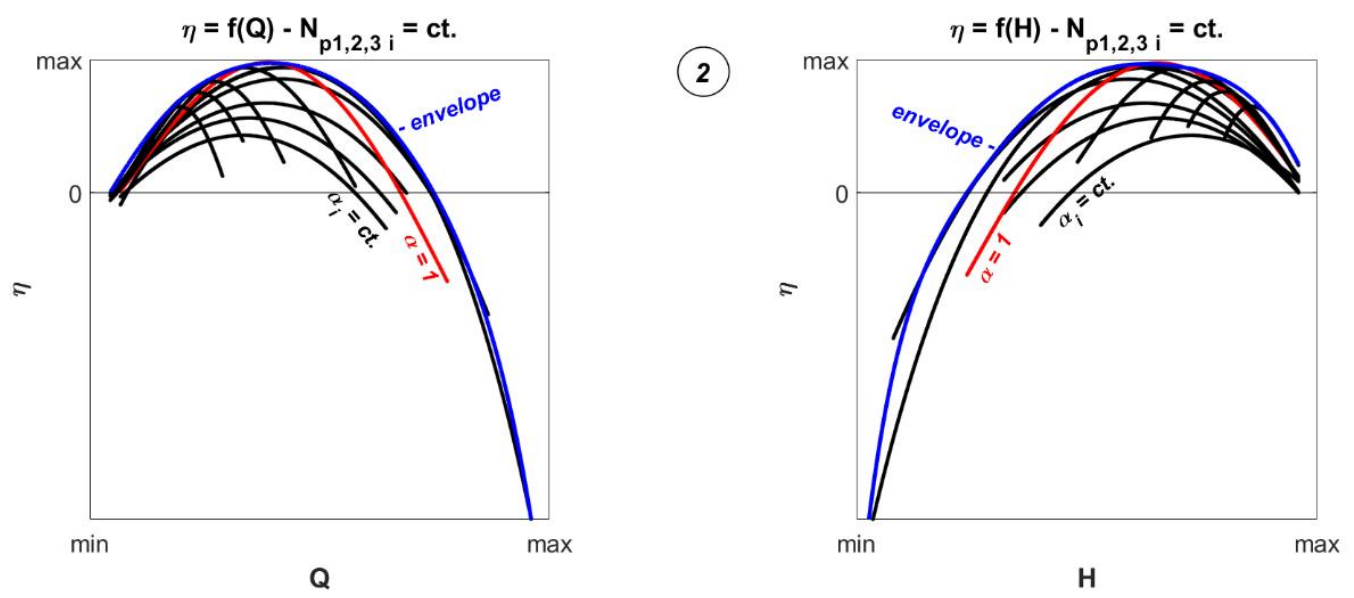

(b)
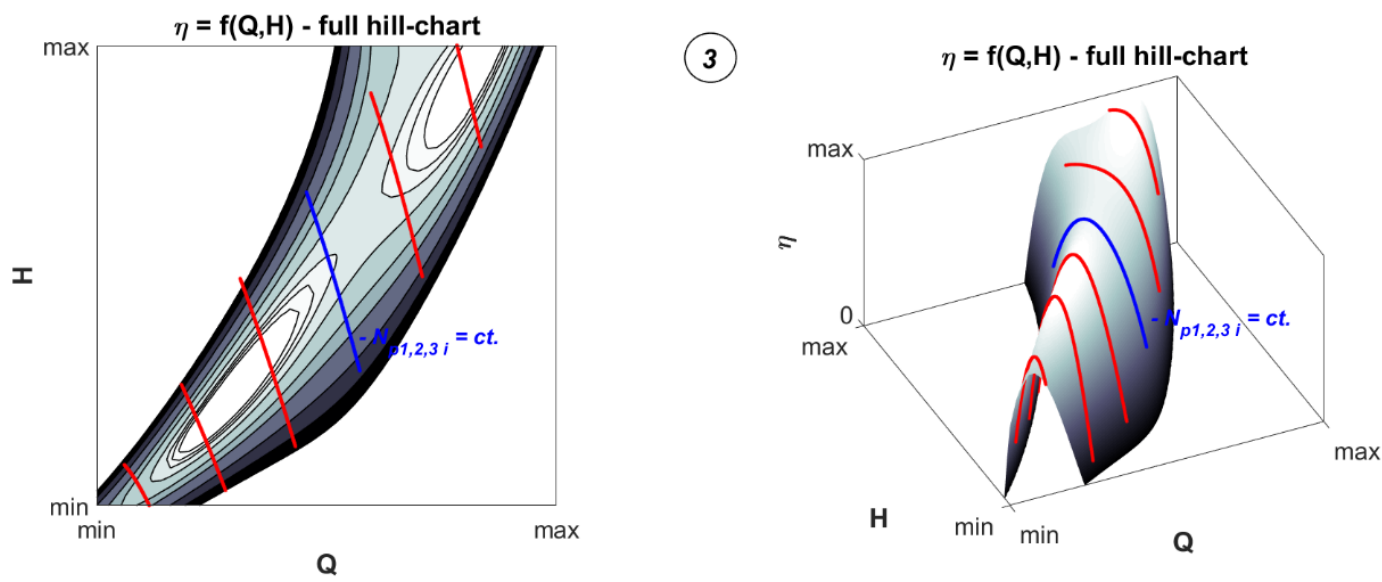

(c)

Figure 2. Employed methodology to reconstruct the full hydraulic efficiency hill-chart of a double-regulated machine using the alternative dynamic measurements method: (a) First step: measurements of several efficiency hill-charts at constant rotational speed $N_{p 1,2,3 i}$ of the test rig recirculating pumps and constant speed ratio between the two runners, $\alpha_{i} ;(b)$ Second step: calculation of discharge-efficiency and head-efficiency envelopes for all testing recirculating pumps' speed values; (c) Third step: construction of the final full 3D hill-chart of the machine by surface interpolation using the previously obtained envelopes.

\section{Experimental Setup}

\subsection{HES-SO VS Hydraulic Testing Infrastructure}

The universal hydraulic test rig of the HES-SO Valais//Wallis-Switzerland (see Figure 3) dedicated to small-power turbomachines has been employed to perform the hydraulic performance measurements. Its configuration, instrumentation and operation follow the IEC 60193 [21] standard recommendations on hydraulic model testing. Three recirculating multistage centrifugal pumps connected in parallel supply the closed-loop circuit of the test rig. The variable-speed pumps $(2 \times 18.5 \mathrm{~kW}$ and respectively $1 \times 5.5 \mathrm{~kW})$ can deliver a maximum discharge of about $100 \mathrm{~m}^{3} / \mathrm{h}$ and a maximum pressure of $160 \mathrm{mWC}$. The testing variable-speed model is placed in the upper part of the circuit, upstream a free-surface pressurized reservoir. The latest allows simulating a given setting level of the model, either positive or negative, and thus investigating also the cavitation performances. The operation of the test rig is ensured by an automatic system. Its customized 
LabVIEW $^{\circledR}$ interface allows for real-time measurements and displays the instantaneous values of pumps' speed, discharge, testing head, water temperature, Thoma number etc. The autonomous regulation system can keep constant the value of the pumps' speeds, the testing head or the discharge. Finally, the wireless communication architecture between the hydraulic test rig and the measurement/monitoring systems (e.g., testing model control system) ensures safe centralization of data, storage and sharing, Hasmatuchi et al. [12].

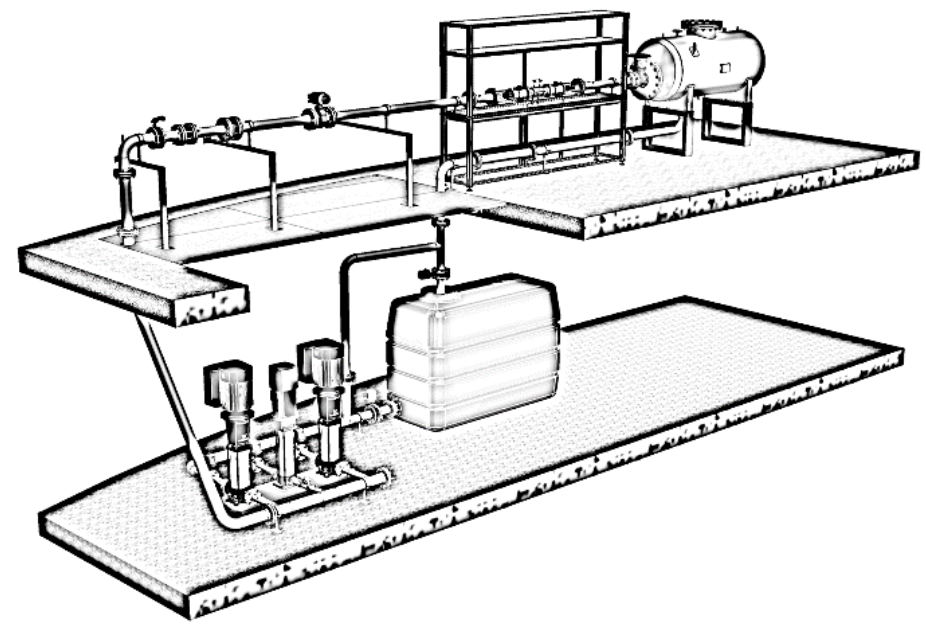

Figure 3. Hydraulic test rig (2016 version) of the HES-SO Valais//Wallis-Switzerland [12]. (Main characteristics: (1) Maximum head: $160 \mathrm{mWC}$; (2) Maximum discharge: $\pm 100 \mathrm{~m}^{3} / \mathrm{h}$; (3) Generating power: $20 \mathrm{~kW}$; (4) Pumping power: $2 \times 18.5 \mathrm{~kW}$ and $1 \times 5.5 \mathrm{~kW}$; (5) Maximum pumps' speed: $3500 / 3000 \mathrm{~min}^{-1}$; (6) Total circuit volume: $4.5 \mathrm{~m}^{3}$ ).

\subsection{Case Studies}

\subsubsection{Microturbine with Counter-Rotating Runners-Bulb Version}

The first case study consists of a fully instrumented laboratory prototype of an axial microturbine with counter-rotating runners (see Figure 4) dedicated to recover the energy dissipated by release valves of water supply networks. Its multi-stage concept, similar to the one of centrifugal pumps, makes it appropriate for high head operating conditions, specific to the Pelton turbines. Indeed, each stage, composed by two counter-rotating axial runners, generally used for low head and large discharge conditions, recovers a fraction of the total head. The one-stage turbine of $2.65 \mathrm{~kW}$ is composed by two counter-rotating runners with 5 and 7 blades respectively. The outer diameter of the runners, as well as the pipe diameter, is $\phi 100 \mathrm{~mm}$; the turbine inner diameter is $\phi 80 \mathrm{~mm}$. The tested version includes a band, installed at the periphery of each runner, provided with a labyrinth in order to limit the leakage between the tip of the blades and the outer fixed wall. At the nominal operating point (a discharge of $37.5 \mathrm{~m}^{3} / \mathrm{h}$ and a head of $20 \mathrm{mWC}$ ), for a ratio $\alpha=\mathrm{N}_{\mathrm{B}} / \mathrm{N}_{\mathrm{A}}=1$ between the runners absolute rotational speed, its hydraulic efficiency, obtained by numerical simulation, reaches 85\% (Biner et al. [22], Münch-Alligné et al. [9]). The optimal operation of the turbine is ensured by the relative rotational speed between the two runners along with their absolute rotational speed. Further, the runners are driven by two independent electrical generators specially designed for this turbine, Melly et al. [23], placed into the upstream and downstream bulbs respectively. Two frequency converters are used to drive the variable-speed electrical generators at the desired constant rotational speed value, whatever the sign of the mechanical torque. Finally, for each runner, an incremental encoder, used mainly for the rotational speed driving, along with a torque meter ensure the mechanical power measurements. A sealed magnetic coupling separates the wet and the dry regions of the machine to protect the embedded instruments. 


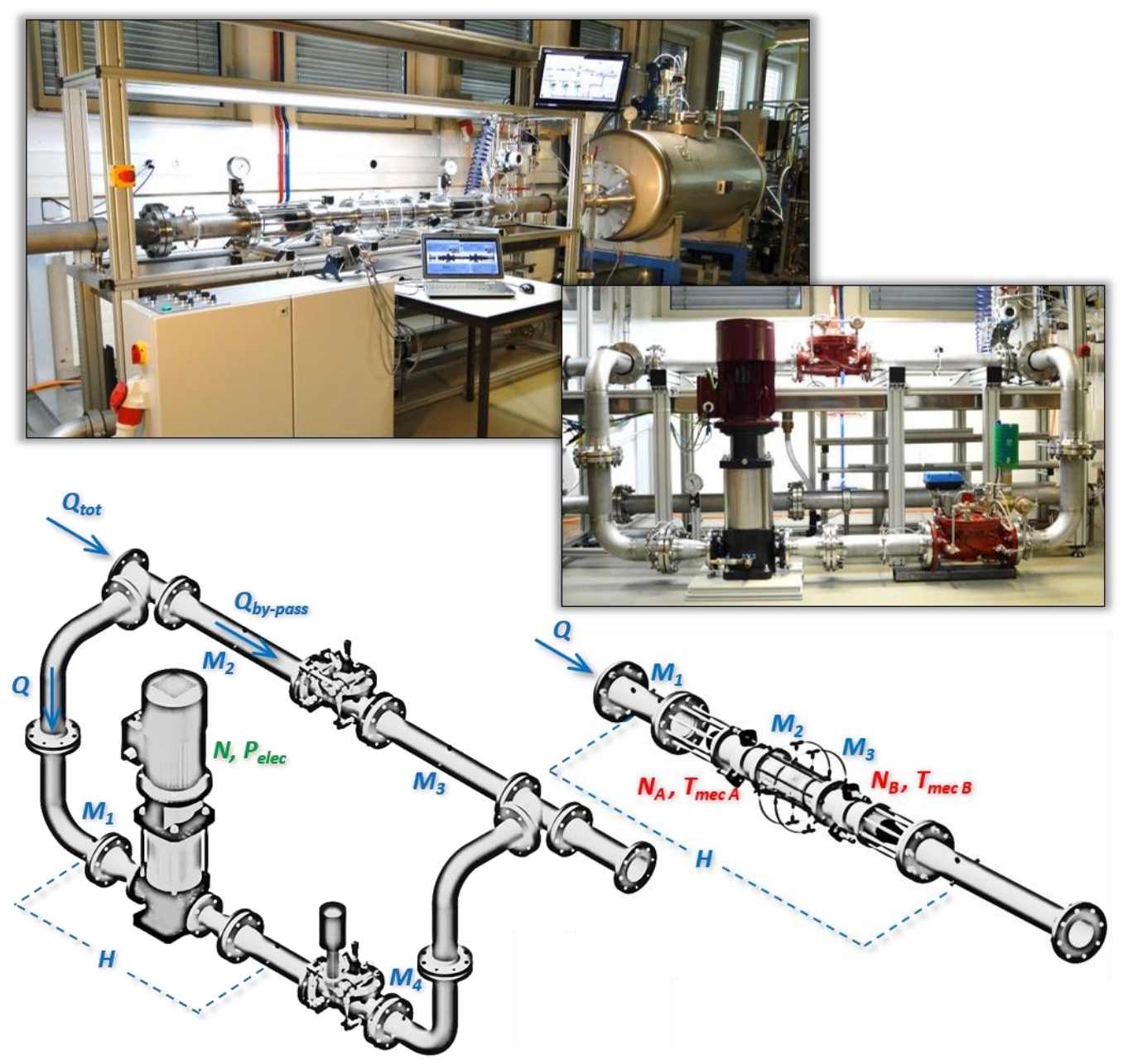

Figure 4. Experimental setup of the counter-rotating microturbine and of the pump-as-turbine (PAT) for hydraulic performance measurements on the test rig.

\subsubsection{Multi-Stage Centrifugal Pump-as-Turbine (PAT)}

Running a standard PAT is often a cost-efficient solution to recover the extra available energy of the water supply systems, and not only-Orchard and Klos [24], Williams [25], Ramos and Borga [26], Carravetta et al. [27] and Motwani et al. [28]. However, prior to the installation, its operation and control in turbine mode must be investigated, Garay [29]. Indeed, if the hydraulic characteristics may be derived using, for example, the experimental data provided by Chapallaz et al. [30], or the method presented by Derakhshan and Nourbakhsh [31], the hydrodynamic stability mainly outside the normal operating range remains difficult or even impossible to predict without experimental tests.

In this context, a second case study dedicated to the energy recovery in water supply networks, consisting of an $11 \mathrm{~kW}$ multi-stage centrifugal pump used as turbine (Ebara EVMG32 5-0F5/11, Tokyo, Japan) has been considered (see Figure 4). Its five radial-axial runners (with 5 blades each) have an inlet diameter of $\phi 136 \mathrm{~mm}$ and an outlet diameter of $\phi 67.5 \mathrm{~mm}$. The PAT is regulated only with the help of the variable rotational speed of the runners. The permanent magnet synchronous generator, Leroy-Sommer LSRPM 132 M (Angoulême, French), with a nominal power of $15.8 \mathrm{~kW}$, has been driven, in this case as well, by a frequency converter able to keep constant the desired rotational speed value, whatever the sign of the mechanical torque or the rotational speed. Concerning the instrumentation, the machine is equipped only with an incremental encoder, necessary for the rotational speed driving. Therefore, for this case, only the electrical power of the generator has been measured. 
One may state here that the PAT has been tested in a hydraulic configuration similar to the one of the pilot site, where it has been already installed. The release valve installed on the by-pass branch, which plays the role of an existing release valve on a drinking water supply network, ensures the extra flow passage when the requested discharge is larger than the maximum flowrate of the turbine. The second release valve, placed downstream the turbine, limits the pressure at the outlet of the main branch, and therefore secures the network, whatever the operating conditions of the turbine. Moreover, in the case of discharges lower than the minimum value allowed for the PAT, or in the case of a failure, this release valve is able to cut completely the main branch, the whole discharge passing automatically through the by-pass one. Anyway, during the performance tests on the PAT, the by-pass release valve remained closed all the time, while the one of the turbine branch has been kept completely opened.

\subsection{Instrumentation}

The characteristics of the main employed instruments necessary to recover the hydraulic performances of the testing model along with the testing conditions are provided in Table 1 . On the one hand, the test rig is equipped with an electromagnetic flowmeter for the discharge $Q$, two differential pressure transducers for the head $\mathrm{H}$ and the setting level $\mathrm{H}_{\mathrm{S}}$ respectively and four additional capacitive absolute pressure transducers for the static pressure at the wall $\mathrm{M}_{1,2,3,4}$. The latest are connected through wall static pressure collectors, as may be seen in Figure 4. A PT100 transducer for the water temperature $\mathrm{T}$ and three optical tachometers for the rotational speed of the recirculation pumps $\mathrm{N}_{\mathrm{p} 1,2,3}$ come to complete the list of instruments.

On the other hand, the axial microturbine is equipped with two incremental encoders and two torque meters, one for each counter-rotating runner, necessary for the driving of the electrical generators and for the computation of the mechanical power. Finally, an incremental encoder and a high-precision electrical multimeter are used to drive the PAT's electrical generator and to compute the electrical power.

Regarding the measurement of quantities related to the test rig (including the hydraulic power), a National Instruments (NI) CompactRIO 9074 autonomous digitizer, equipped with various acquisition/control modules, is used. A NI cDAQ 9174 digitizer is then employed to drive the testing model and to record the values of the rotational speed of the runners, of the mechanical torque of the runners (microturbine) and of the electrical power of the generator (PAT).

In the case of classical static point-by-point method, both digitizers perform measurements and compute the average and the standard deviation values for all parameters over $8 \mathrm{~s}$ at $50 \mathrm{~Hz}$ (user-configurable, depending on the stability of the operating condition). In the case of dynamic measurements, a second NI CDAQ-9174 digitizer has been installed to acquire synchronized dynamic signals of the sensors in parallel with the existing acquisition/control systems of the test rig and of the testing model. Its acquisition frequency has been set to $100 \mathrm{~Hz}$, considered fast enough to cope with the frequency response of the employed sensors. 
Table 1. Main characteristics of the employed instruments for performance measurements.

\begin{tabular}{llll}
\hline Measured Quantity & Sensor Type & Range & Accuracy \\
\hline Discharge $(\mathrm{Q})$ & $\begin{array}{l}\text { Danfoss MAGFLO MAG3100 Water } \\
\text { electromagnetic flowmeter }\end{array}$ & $0 . . \pm 100\left[\mathrm{~m}^{3} / \mathrm{h}\right]$ & $\pm 0.5[\%]$ \\
\hline Head (H) & $\begin{array}{l}\text { Endress and Hauser Deltabar M PMD55 } \\
\text { differential pressure sensor }\end{array}$ & $0 . .16[\mathrm{bar}]$ & $\pm 0.1[\%]$ \\
\hline Setting level $\left(\mathrm{H}_{\mathrm{S}}\right)$ & Fuji FCX-CII Series differential pressure sensor & $0 . .5[\mathrm{bar}]$ & $\pm 0.2[\%]$ \\
\hline Absolute static pressure $\left(\mathrm{M}_{1,2,3,4}\right)$ & $\begin{array}{l}\text { Endress and Hauser Cerabar T PMC 131 } \\
\text { capacitive pressure transducer }\end{array}$ & $0 . .10 / 20[\mathrm{bar}]$ & $\pm 0.05[\%]$ \\
\hline Water Temperature (T) & $\begin{array}{l}\text { Endress and Hauser Easytemp TMR 31 PT100 } \\
\text { transducer }\end{array}$ & $0 . .100\left[{ }^{\circ} \mathrm{C}\right]$ & $\pm 0.1[\%]$ \\
\hline Pumps' rotational speed $\left(\mathrm{N}_{\mathrm{p} 1,2,3}\right)$ & $\begin{array}{l}\text { Sick VTF180-2P42417 photoelectric proximity } \\
\text { sensor }\end{array}$ & $0 . .1000[\mathrm{~Hz}]$ & - \\
\hline Mechanical torque ( $\left.\mathrm{T}_{\mathrm{mec}} \mathrm{A}, \mathrm{B}\right)$ & NCTE 2200 torquemeter & $0 . . \pm 7.5[\mathrm{Nm}]$ & $\pm 1[\%]$ \\
\hline Turbine rotational speed $\left(\mathrm{N}_{\mathrm{A}, \mathrm{B}}\right)$ & IED incremental encoder & $0 . .7500\left[\mathrm{~min}^{-1}\right]$ & $2048[\mathrm{ppr}]$ \\
\hline Electrical power $\left(\mathrm{P}_{\text {elec }}\right)$ & $\begin{array}{l}\text { Zimmer LMG500 precision electrical } \\
\text { multimeter }\end{array}$ & $\begin{array}{l}0 . .1000\left[\mathrm{~V}_{\text {trms }}\right] \\
0 . .32\left[\mathrm{~A}_{\text {trms }}\right]\end{array}$ & $\pm 0.03[\%]$ \\
\hline PAT rotational speed $(\mathrm{N})$ & Incremental encoder & $0 . .6000\left[\mathrm{~min}^{-1}\right]$ & $4096[\mathrm{ppr}]$ \\
\hline Data acquisition and control & Dedicated to the control/monitoring of the test rig & \\
\hline NI CompactRIO 9074 controller & Dedicated to the control/monitoring of the testing model using the standard static \\
\hline NI cDAQ-9174 digitizer & measurements method & Dedicated to the control/monitoring of the testing model for the dynamic measurements \\
\hline NI cDAQ-9174 digitizer &
\end{tabular}

\subsection{Employed Testing Protocol}

The employed experimental protocol to measure the hydraulic performances of both the microturbine and the PAT is provided in Figure 5. First, all the instruments have been systematically calibrated and/or rechecked before the beginning of measurements.

Considering the classical point-by-point method, the measurements on the microturbine have been performed for 11 different testing head values to build the full efficiency 3D hill-chart of the microturbine. Moreover, as resumed in Table 2, nine values of constant ratio $\alpha$ between the runners' absolute rotational speed were systematically considered for each constant testing head. That makes a total of more than 1000 acquired operating points.

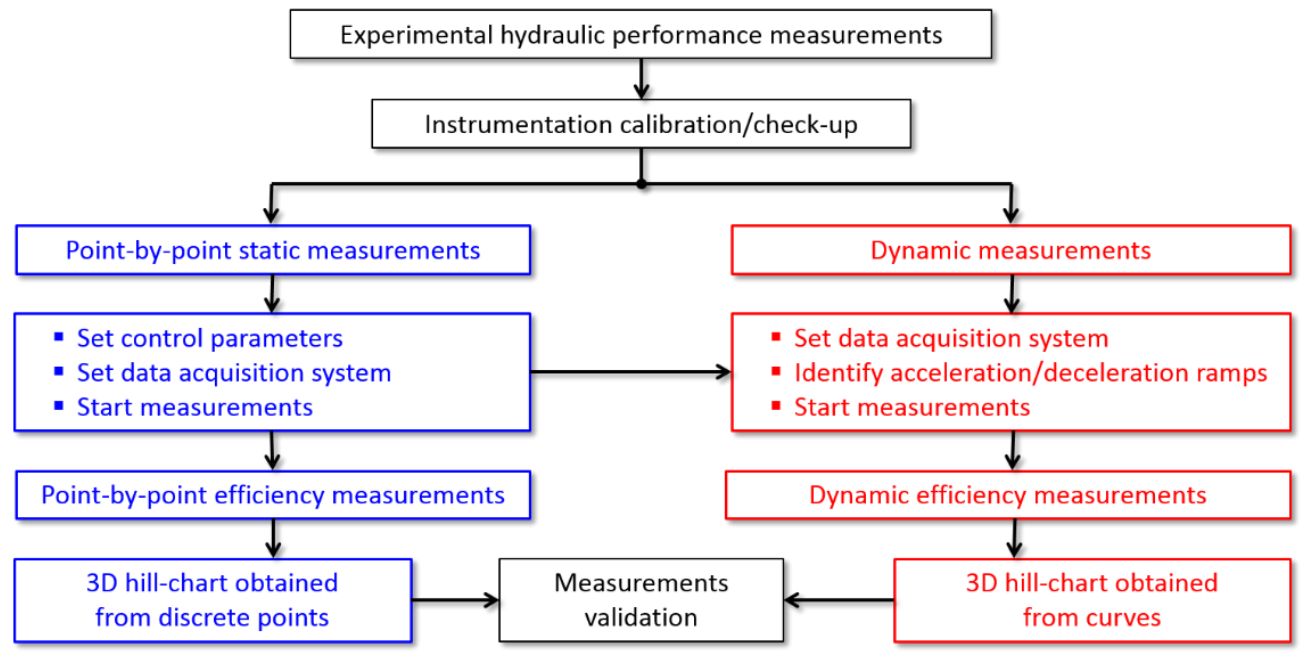

Figure 5. Flowchart of the employed experimental protocol [32,33]. 
Table 2. Combinations of rotational speeds of the microturbine runners for point-by-point performance measurements.

\begin{tabular}{|c|c|c|c|c|c|c|c|c|c|}
\hline \multicolumn{10}{|c|}{$\mathrm{H}=0.5 / 0.75 / 1 / 1.25 / 1.5 / 1.75 / 2 / 2.25 / 2.5 / 2.75 / 3[\mathrm{bar}]$} \\
\hline $\mathbf{N}_{\mathrm{A}}[\%]$ & & & & & $\mathbf{N}_{\mathbf{B}}$ & & & & \\
\hline 0 & 0 & - & - & - & - & - & - & - & - \\
\hline 8.33 & 0 & 2.08 & 4.17 & 6.25 & 8.33 & 11.08 & 16.67 & 33.33 & 8.33 \\
\hline 16.67 & 0 & 4.17 & 8.33 & 12.5 & 16.67 & 22.17 & 33.33 & 66.67 & 16.67 \\
\hline 25 & 0 & 6.25 & 12.5 & 18.75 & 25 & 33.33 & 50 & 100 & 25 \\
\hline 33.33 & 0 & 8.33 & 16.67 & 25 & 33.33 & 44.47 & 66.67 & - & 33.33 \\
\hline 41.67 & 0 & 10.42 & 20.83 & 31.25 & 41.67 & 55.42 & 83.33 & - & 41.67 \\
\hline 50 & 0 & 12.5 & 25 & 37.5 & 50 & 66.67 & 100 & - & 50 \\
\hline 58.33 & 0 & 14.58 & 29.17 & 43.75 & 58.33 & 77.58 & - & - & 58.33 \\
\hline 66.67 & 0 & 16.67 & 33.33 & 50 & 66.67 & 88.9 & - & - & 66.67 \\
\hline 75 & 0 & 18.75 & 37.5 & 56.25 & 75 & 100 & - & - & 75 \\
\hline 83.33 & 0 & 20.83 & 41.67 & 62.5 & 83.33 & - & - & - & 83.33 \\
\hline 91.67 & 0 & 22.92 & 45.83 & 68.75 & 91.67 & - & - & - & 91.67 \\
\hline 100 & 0 & 25 & 50 & 75 & 100 & - & - & - & 100 \\
\hline$\alpha=\mathbf{N}_{\mathbf{B}} / \mathbf{N}_{\mathbf{A}}[-]$ & 0 & 0.25 & 0.5 & 0.75 & 1 & 1.33 & 2 & 4 & $\infty\left(\mathrm{N}_{\mathrm{A}}=0\right)$ \\
\hline
\end{tabular}

In the case of the PAT, the measurements have been performed at 10 different constant rotational speed values of the recirculating pumps. For each recirculating pumps' constant rotational speed value, more than 12 different runner rotational speed values of the PAT have been acquired (see Table 3), which makes a total of more than 120 operating points necessary to draw the full-3D efficiency characteristic of the machine. Compared to the microturbine, the number of operating points is less since its only degree of freedom is the rotational speed of the runner.

Table 3. Combinations of rotational speeds of the recirculating pumps and of the PAT for performance measurements using the static method.

\begin{tabular}{|c|c|c|c|c|c|c|c|c|c|c|}
\hline & \multicolumn{10}{|c|}{$\mathrm{N}_{\mathrm{p} 1,2,3}[\%]$} \\
\hline & 24 & 33.33 & 41.67 & 50 & 58.33 & 66.67 & 75 & 83.33 & 91.67 & 100 \\
\hline \multirow{12}{*}{$\frac{\delta}{\frac{0}{a}}$} & 8.33 & 8.33 & 8.33 & 8.33 & 8.33 & 8.33 & 8.33 & 8.33 & 50 & 78 \\
\hline & 16.67 & 16.67 & 16.67 & 16.67 & 16.67 & 16.67 & 16.67 & 16.67 & 58.33 & 83.33 \\
\hline & 25 & 25 & 25 & 25 & 25 & 25 & 25 & 25 & 66.67 & - \\
\hline & 33.33 & 33.33 & 33.33 & 33.33 & 33.33 & 33.33 & 33.33 & 33.33 & 75 & - \\
\hline & 41.67 & 41.67 & 41.67 & 41.67 & 41.67 & $41.6 \cdot 7$ & 41.67 & 41.67 & 83.33 & - \\
\hline & 50 & - & 50 & 50 & 50 & 50 & 50 & 50 & 91.67 & - \\
\hline & 58.33 & - & 58.33 & 58.33 & 58.33 & 58.33 & 58.33 & 58.33 & 100 & - \\
\hline & - & - & - & 66.67 & 66.67 & 66.67 & 66.67 & 66.67 & - & - \\
\hline & - & - & - & 75 & 75 & 75 & 75 & 75 & - & - \\
\hline & - & - & - & - & 83.33 & 83.33 & 83.33 & 83.33 & - & - \\
\hline & - & - & - & - & 91.67 & 91.67 & 91.67 & 91.67 & - & - \\
\hline & - & - & - & - & - & 100 & 100 & 100 & - & - \\
\hline
\end{tabular}

Focusing on the dynamic method, first the optimal acceleration/deceleration ramps of the electrical drives have been identified in order to ensure a quasi- steady-state operation of the testing model. The main objective was to reduce at maximum the measurement time, while avoiding measurement errors and hysteresis of the acquired characteristics. Then, the hydraulic efficiency measurements have been performed at respectively 9 and 19 different test rig recirculating pumps' constant rotational speed values (see Tables 4 and 5) while increasing and decreasing the speed of the microturbine/PAT runner(s) from minimum to maximum, and vice versa. In the case of the microturbine, for each recirculating pumps' constant rotational speed value 11 different constant runners absolute rotational speed ratios have been tested. Finally, the results obtained by the dynamic method have been validated with the ones obtained by the classical static point-by-point measurements method. 
Table 4. Microturbine runners rotational speed variation ranges for performance measurements using the dynamic method.

\begin{tabular}{|c|c|c|c|c|c|c|c|c|c|c|}
\hline \multicolumn{11}{|c|}{$\mathrm{N}_{\mathrm{p} 1,2,3}[\%]$} \\
\hline & 24 & 33.33 & 41.67 & 50 & 58.33 & 66.67 & 75 & 83.33 & 91.67 & $\alpha=\mathbf{N}_{\mathrm{B}} / \mathbf{N}_{\mathrm{A}}[-]$ \\
\hline \multirow{10}{*}{$\frac{\bar{o}}{z^{\circ}}$} & $0 \div 100$ & $0 \div 100$ & $0 \div 100$ & $0 \div 100$ & $0 \div 100$ & $33.33 \div 100$ & $75 \div 100$ & $83.33 \div 100$ & $91.67 \div 100$ & 0 \\
\hline & $0 \div 100$ & $0 \div 100$ & $0 \div 100$ & $0 \div 100$ & $41.67 \div 100$ & - & - & - & - & 0.125 \\
\hline & $0 \div 100$ & $0 \div 100$ & $0 \div 100$ & $0 \div 100$ & $41.67 \div 100$ & $83.33 \div 100$ & - & - & - & 0.25 \\
\hline & $0 \div 100$ & $0 \div 100$ & $0 \div 100$ & $0 \div 100$ & $33.33 \div 100$ & $58.33 \div 100$ & $91.67 \div 100$ & - & - & 0.5 \\
\hline & $0 \div 100$ & $0 \div 100$ & $0 \div 100$ & $0 \div 100$ & $25 \div 100$ & $50 \div 100$ & $75 \div 100$ & - & - & 0.75 \\
\hline & $0 \div 100$ & $0 \div 100$ & $0 \div 100$ & $0 \div 100$ & $25 \div 100$ & $41.67 \div 100$ & $58.33 \div 100$ & $83.33 \div 100$ & - & 1 \\
\hline & $0 \div 75$ & $0 \div 75$ & $0 \div 75$ & $0 \div 75$ & $18.75 \div 75$ & $31.25 \div 75$ & $50 \div 75$ & - & - & 1.33 \\
\hline & $0 \div 50$ & $0 \div 50$ & $0 \div 50$ & $0 \div 50$ & $12.5 \div 50$ & $25 \div 50$ & $45.83 \div 50$ & - & - & 2 \\
\hline & $0 \div 25$ & $0 \div 25$ & $0 \div 25$ & $0 \div 25$ & $6.25 \div 25$ & $12.5 \div 25$ & - & - & - & 4 \\
\hline & $0 \div 12.5$ & $0 \div 12.5$ & $0 \div 12.5$ & $0 \div 12.5$ & $4.17 \div 12.5$ & - & - & - & - & 8 \\
\hline$\frac{\overline{0}}{\overbrace{}^{\infty}}$ & $0 \div 100$ & $0 \div 100$ & $0 \div 100$ & $0 \div 100$ & $33.33 \div 100$ & - & - & - & - & $\infty\left(\mathrm{N}_{\mathrm{A}}=0\right)$ \\
\hline
\end{tabular}

Table 5. Combinations of rotational speeds of the recirculating pumps and of the PAT for performance measurements using the dynamic method.

\begin{tabular}{ccccccccccc}
\hline \multirow{2}{*}{$\mathrm{N}_{\mathrm{p} 1,2,3}[\%]$} & 24 & 29.17 & 33.33 & 37.5 & 41.67 & 45.83 & 50 & 54.17 & 58.33 & 62.5 \\
\cline { 2 - 10 } & 66.67 & 70.83 & 75 & 79.17 & 83.33 & 87.5 & 91.67 & 95.83 & 100 & \\
\hline $\mathrm{N}_{\text {PAT }}[\%]$ & & & $8.33 \div 100$ & & & & \\
\hline
\end{tabular}

\section{Results}

The hydraulic-to-electrical efficiency $\eta_{\text {h-elec }}$ can be expressed as the product between the hydraulic-to-mechanical efficiency $\eta_{\mathrm{h}-\mathrm{mec}}$ and the electrical efficiency of the generator $\eta_{\text {elec }}$ :

$$
\eta_{\mathrm{h}-\text { elec }}=\eta_{\mathrm{h}-\text { mec }} \times \eta_{\text {elec }}=\eta_{\mathrm{h}} \times \eta_{\mathrm{m}} \times \eta_{\text {elec }}=\left(\eta_{\mathrm{e}} \times \eta_{\mathrm{q}} \times \eta_{\mathrm{rm}}\right) \times \eta_{\mathrm{m}} \times \eta_{\text {elec }}[\%]
$$

Further, $\eta_{\mathrm{h}-\mathrm{mec}}$ results from the product between the hydraulic $\eta_{\mathrm{h}}$ and the bearing efficiency $\eta_{\mathrm{m}}$, with the hydraulic efficiency $\eta_{\mathrm{h}}$ including the efficiency of the disc friction $\eta_{\mathrm{rm}}$, the energetic efficiency $\eta_{\mathrm{e}}$ as well as the volumetric efficiency $\eta_{\mathrm{q}}$. In the case of the microturbine, considering its reduced size and geometrical complexity, only the hydraulic-to-mechanical efficiency $\eta_{\mathrm{h}-\mathrm{m}}$, given by the ratio between the mechanical power $P_{\text {mec }}$ recovered by both runners and the hydraulic power $P_{h}$ of the whole one-stage turbine, could be measured:

$$
\eta_{\mathrm{h}-\mathrm{mec}}=\frac{\mathrm{P}_{\mathrm{mec}}}{\mathrm{P}_{\mathrm{h}}}=\frac{\sum\left(\omega_{\mathrm{i}} \times \mathrm{T}_{\text {mec i }}\right)}{\rho \times \mathrm{Q} \times \mathrm{E}}[\%]
$$

with

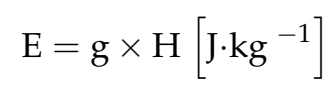

In the case of the PAT, since the machine is not equipped with a torquemeter, only the hydraulic-to-electrical efficiency, given by the ration between the electrical power of the generator $P_{\text {elec }}$ and the hydraulic power has been measured:

$$
\eta_{\text {h-elec }}=\frac{P_{\text {elec }}}{P_{h}}=\frac{P_{\text {elec }}}{\rho \times Q \times E}[\%]
$$

Regarding the hydraulic power, whilst the discharge $Q$ is directly measured, the total specific energy $\mathrm{E}$ is calculated with the value of the head H. As illustrated in Figure 4, for both tested cases, the inline inlet and outlet cross sections of the machine are equal. Considering the Bernoulli's equation, for this particular case the calculation of the total head can be performed only from the difference of the static pressure between the inlet and the outlet, directly measured with the help of the differential pressure sensor. 


\subsection{Influence of the Runner Speed Acceleration/Deceleration Ramp}

The first step before performing dynamic measurements is to find the optimal acceleration/deceleration ramp of the runner(s) speed in order to stay in quasi- steady-state operating condition during the measurement process. To this end, the speed of the three recirculating pumps of the test rig has been set and maintained constant at a value of $1500 \mathrm{~min}^{-1}$ in the case of the microturbine, or $2000 \mathrm{~min}^{-1}$ in the case of the PAT. Then, for the microturbine, considering a constant runners absolute rotational speed ratio $\alpha=1$, the runners speed was uniformly increased from $1000 \mathrm{~min}^{-1}$ to $2000 \mathrm{~min}^{-1}$ and then decreased back to $1000 \mathrm{~min}^{-1}$. The same procedure was applied in the case of the PAT for the range from $750 \mathrm{~min}^{-1}$ to $2250 \mathrm{~min}^{-1}$. In total, 6 (for the microturbine) and respectively 4 (for the PAT) different acceleration/deceleration ramps of respectively 10, 25, 30, 40, 60,90 and $120 \mathrm{~s} / 1000 \mathrm{~min}^{-1}$ have been addressed.

$$
\begin{gathered}
\eta^{*}=\frac{\eta_{\mathrm{h}-\text { mec }} / \mathrm{h}-\text { elec }}{\max \left(\eta_{\mathrm{h}-\text { mec }} / \mathrm{h} \text {-elec }\right)}[-] \\
\eta^{* \prime}=\eta^{*}-\eta_{\text {fit }}^{*}[-]
\end{gathered}
$$

In Figure 6, the resulting influence of the acceleration/deceleration ramp of the runner(s) speed on power and on the efficiency, for fixed inflow conditions, is provided. The efficiency is scaled with the maximum measured value determined from the static measurements (see Equation (5)). One may notice here hysteresis and large measurement errors on the resulting efficiency for speed ramps below $40 \mathrm{~s} / 1000 \mathrm{~min}^{-1}$.

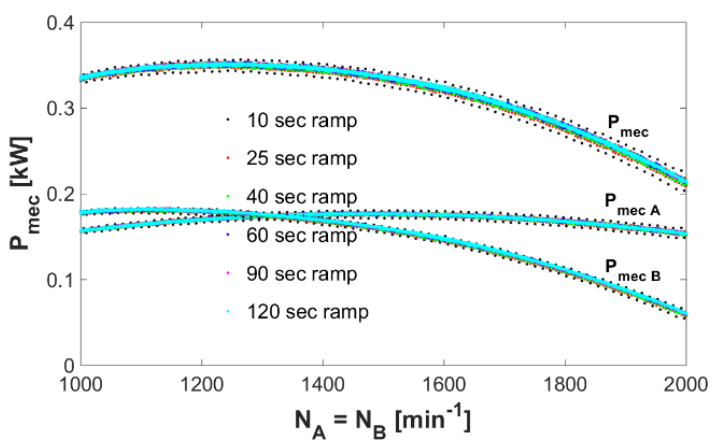

(a)

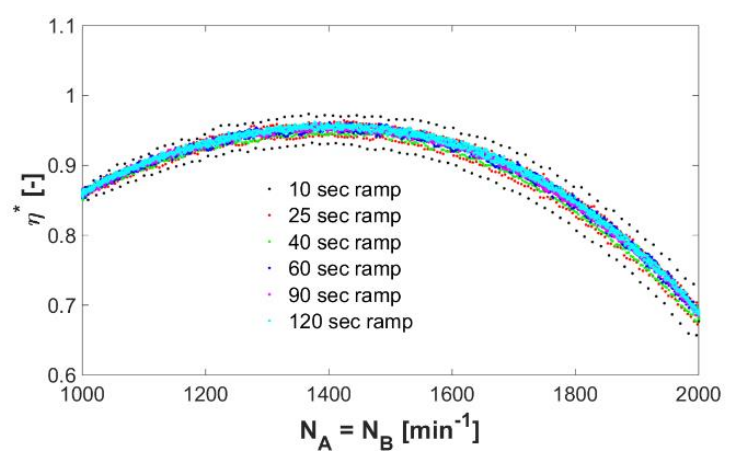

(c)

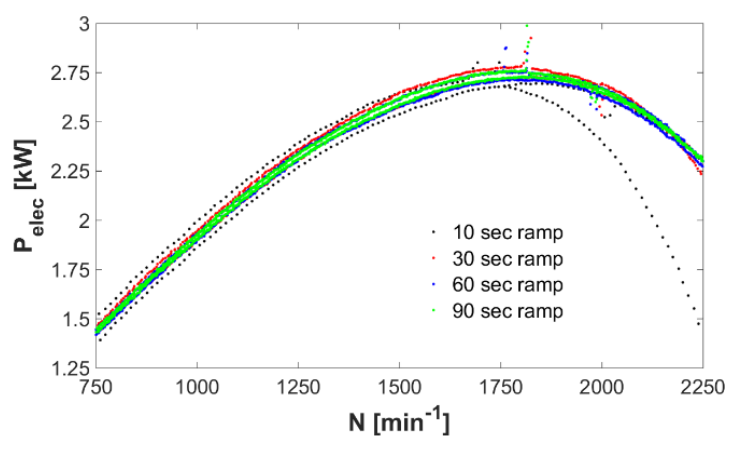

(b)

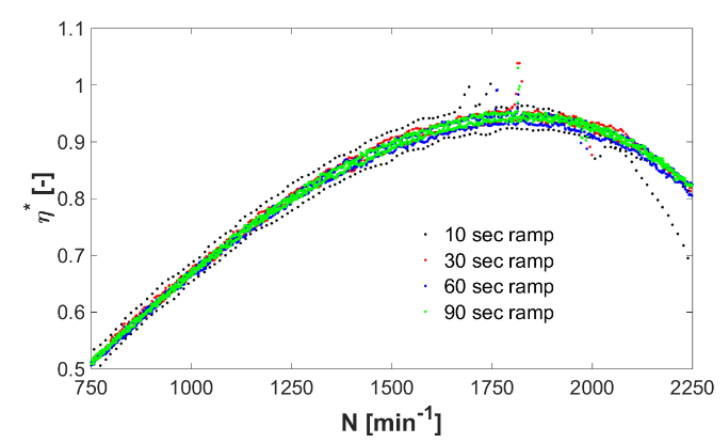

(d)

Figure 6. Influence of the acceleration/deceleration ramp of the runner(s) speed on the power and on the efficiency during one forth and back cycle at fixed testing inflow conditions: (a) variation of the counter-rotating microturbine mechanical power $\left(\mathrm{N}_{\mathrm{p} 1,2,3}=1500 \mathrm{~min}^{-1}\right)$; (b) variation of the PAT electrical power $\left(\mathrm{N}_{\mathrm{p} 1,2,3}=2000 \mathrm{~min}^{-1}\right)$; (c) variation of the counter-rotating microturbine efficiency $\left(\mathrm{N}_{\mathrm{p} 1,2,3}=1500 \mathrm{~min}^{-1}\right) ;(\mathbf{d})$ variation of the PAT efficiency $\left(\mathrm{N}_{\mathrm{p} 1,2,3}=2000 \mathrm{~min}^{-1}\right)$. 
Then, the Figure 7 presents the influence of the speed acceleration/deceleration ramp on the magnitude of the efficiency fluctuations (due to measurement errors) $\eta^{* \prime}$ compared to the average efficiency obtained by curve fitting (see Equation (6)). Accordingly, for both tested cases, the hysteresis effect tends to become insignificant for speed ramps larger than $40 \mathrm{~s} / 1000 \mathrm{~min}^{-1}$. The evolution of the resulting standard deviation (STD) of the efficiency fluctuation $\eta^{* \prime}$ STD with the speed ramp, provided in Figure 8 and Table 6 confirms this assertion. Consequently, as an acceptable compromise between the measurement time and the measurement precision (efficiency errors below $1 \%$ for both cases) a speed acceleration/deceleration ramp of $60 \mathrm{~s} / 1000 \mathrm{~min}^{-1}$ has been selected for the further dynamic efficiency measurements.

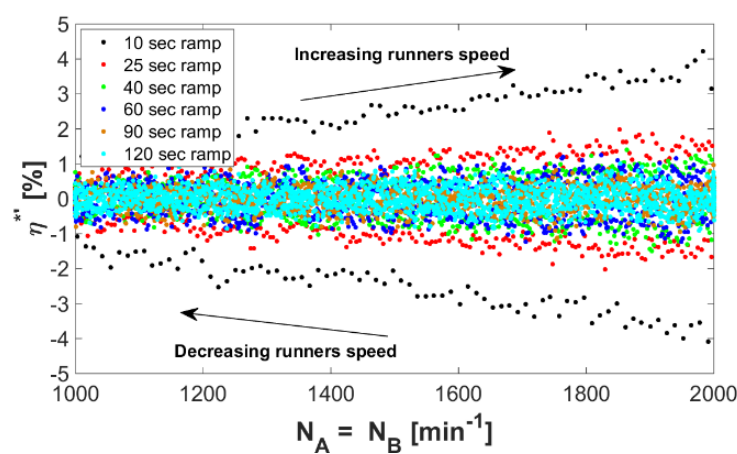

(a)

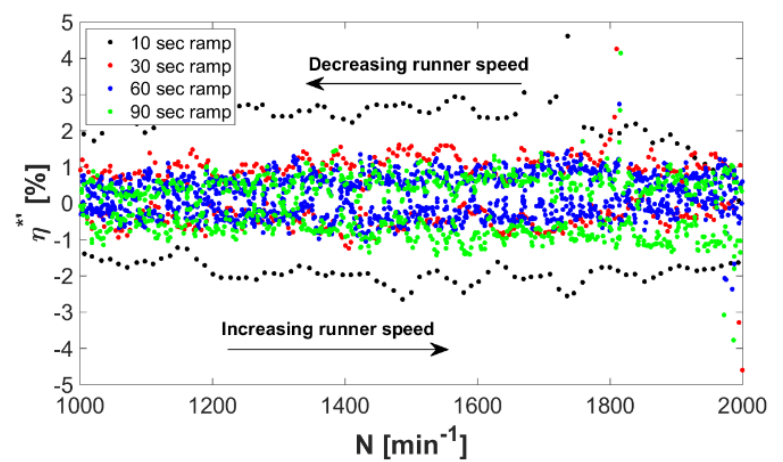

(b)

Figure 7. Influence of the acceleration/deceleration ramp of the runner(s) speed on the efficiency fluctuation during one forth and back cycle at fixed testing inflow conditions: (a) counter-rotating microturbine-constant test rig pumps' speed of $\mathrm{N}_{\mathrm{p} 1,2,3}=1500 \mathrm{~min}^{-1}$; (b) PAT—constant test rig pumps' speed of $\mathrm{N}_{\mathrm{p} 1,2,3}=2000 \mathrm{~min}^{-1}$.

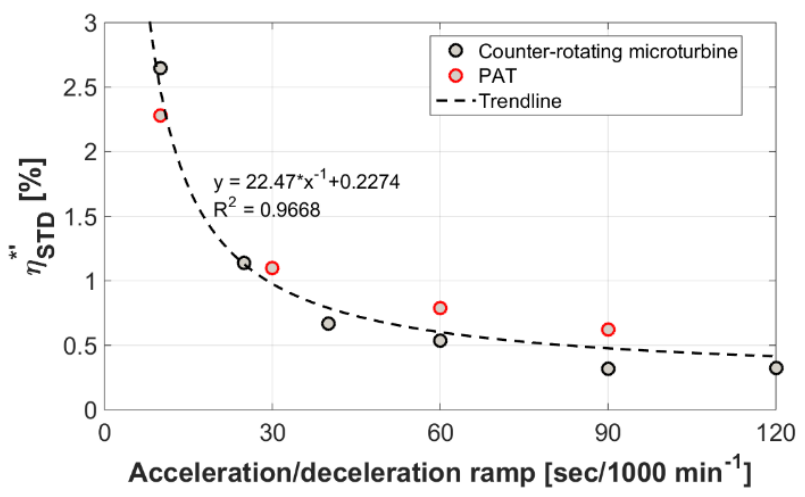

Figure 8. Resulting efficiency fluctuation standard deviation (STD) depending on the acceleration/deceleration ramp. 
Table 6. Resulting STD values of the efficiency fluctuation depending on the acceleration/deceleration ramp.

\begin{tabular}{ccc}
\hline \multirow{2}{*}{$\begin{array}{c}\text { Acceleration/Deceleration Ramp } \\
\text { [s/1000 } \text { min }^{-1} \text { ] }\end{array}$} & \multicolumn{2}{c}{$\eta^{\text {*'s } \text { STD [\%] }}$} \\
\cline { 2 - 3 } & Microturbine & PAT \\
\hline 10 & 2.6467 & 2.2774 \\
25 & 1.1363 & - \\
30 & - & 1.0995 \\
40 & 0.6693 & - \\
60 & 0.5369 & 0.7863 \\
90 & 0.3167 & 0.6246 \\
120 & 0.3213 & - \\
\hline
\end{tabular}

\subsection{Dynamic versus Static Measurements Results and Validation}

\subsubsection{Results at Fixed Testing Inflow Conditions}

The comparison on the power coefficient and on the efficiency between the values obtained by dynamic and by static point-by-point measurement methods, for fixed inflow conditions (recirculating pumps' speed of $\mathrm{N}_{\mathrm{p}}=1500 / 2000 \mathrm{~min}^{-1}$ ), is given in Figure 9. In the case of the discrete point-by-point method, the speed of the turbine runner(s) has been modified in steps of $250 \mathrm{~min}^{-1}$ from 0 to $3000 \mathrm{~min}^{-1}$, whilst is the case of the dynamic method, it has been continuously increased, and then decreased, from 0 to $3000 \mathrm{~min}^{-1}$, and vice versa. A good agreement between the results obtained by the two methods, with a maximum error of $1 \%$ between the mean values, may be observed for both tested cases, overall operating range, including off-design conditions. In the case of the microturbine, the negative efficiency values are given by a negative mechanical torque of the runners, corresponding to the turbine brake operating mode.

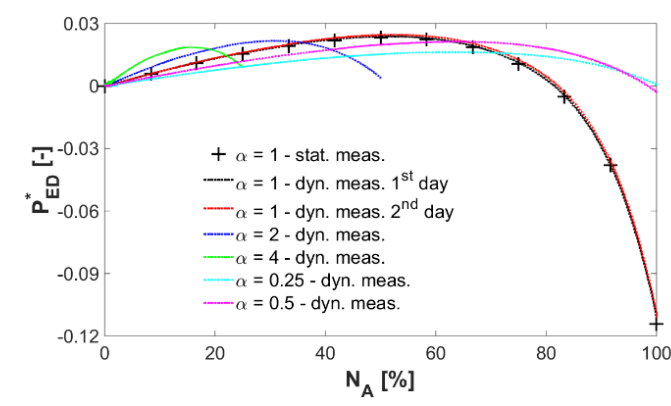

(a)

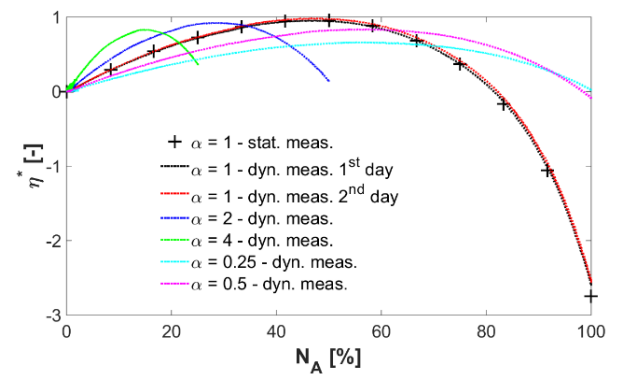

(c)

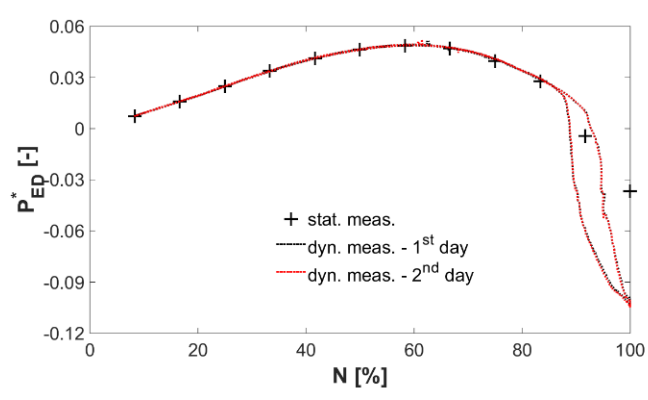

(b)

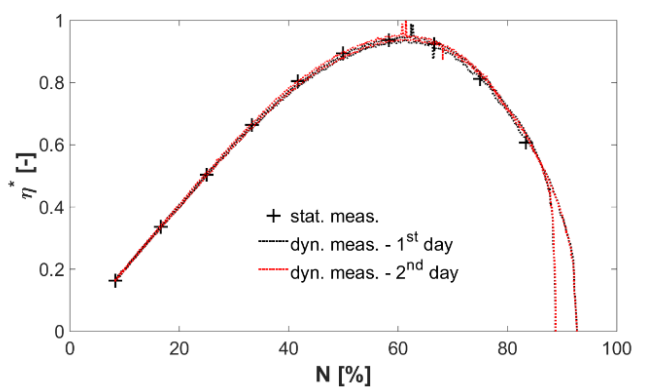

(d)

Figure 9. Dynamic versus static power coefficient and efficiency measurements during one forth and back cycle at fixed testing inflow conditions: (a) variation of the counter-rotating microturbine power coefficient $\left(\mathrm{N}_{\mathrm{p} 1,2,3}=1500 \mathrm{~min}^{-1}\right)$; (b) variation of the PAT power coefficient $\left(\mathrm{N}_{\mathrm{p} 1,2,3}=2000 \mathrm{~min}^{-1}\right)$; (c) variation of the counter-rotating microturbine efficiency $\left(\mathrm{N}_{\mathrm{p} 1,2,3}=1500 \mathrm{~min}^{-1}\right)$; (d) variation of the PAT efficiency $\left(\mathrm{N}_{\mathrm{p} 1,2,3}=2000 \mathrm{~min}^{-1}\right)$. 


\subsubsection{Resulting PAT 4-Quadrant Characteristic}

In the case of the PAT, an accentuated hysteresis, specific to S-shaped characteristic curves, is noticed for high rotational speed values. To this end, considering the speed, the discharge and the power factors (Equations (7)-(9)), the resulting S-shaped 4-quadrants characteristic curves of the PAT measured through the dynamic method are provided in Figure 10. Focusing on the discharge-speed characteristic, all the measurements are clustered on only one S-shaped curve. Indeed, this is explained by the fact that the machine has not been designed with any distributor, its characteristic being similar to the one of a pump-turbine at fixed guide vanes opening angle. However, on a power-speed representation, depending on the recirculating pumps' speed, several S-shaped characteristics and hysteresis are noticed. Concerning the hydraulic configuration of the test rig for these 4-quadrants measurements, no special adjustment has been applied, the machine running smoothly between the turbine and reverse-pump modes, and vice versa.

$$
\begin{gathered}
\mathrm{N}_{\mathrm{ED}}=\frac{\mathrm{N} \times \mathrm{D}_{\text {ref }}}{60 \times \sqrt{\mathrm{E}}}[-] \\
\mathrm{Q}_{\mathrm{ED}}=\frac{\mathrm{Q}}{\mathrm{D}_{\mathrm{ref}}^{2} \sqrt{\mathrm{E}}}[-] \\
\mathrm{P}_{\mathrm{ED}}^{*}=\frac{P_{\mathrm{mec} / \text { elec }}}{\rho \times \mathrm{D}_{\mathrm{ref}}^{2} \mathrm{E}^{1.5}}[-]
\end{gathered}
$$

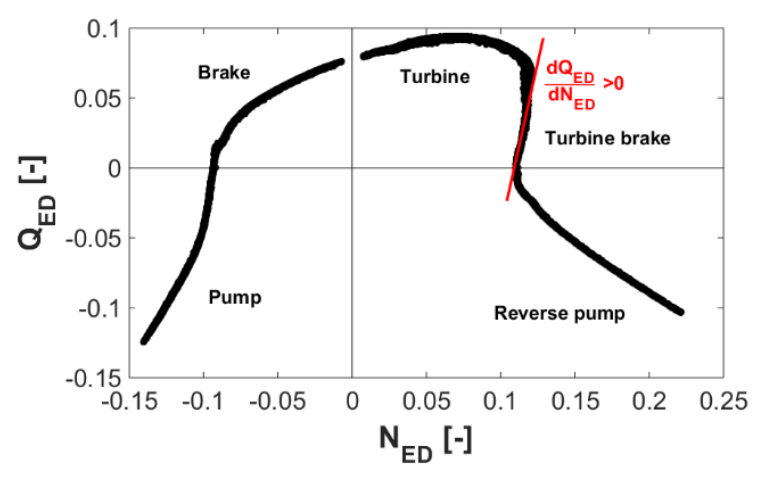

(a)

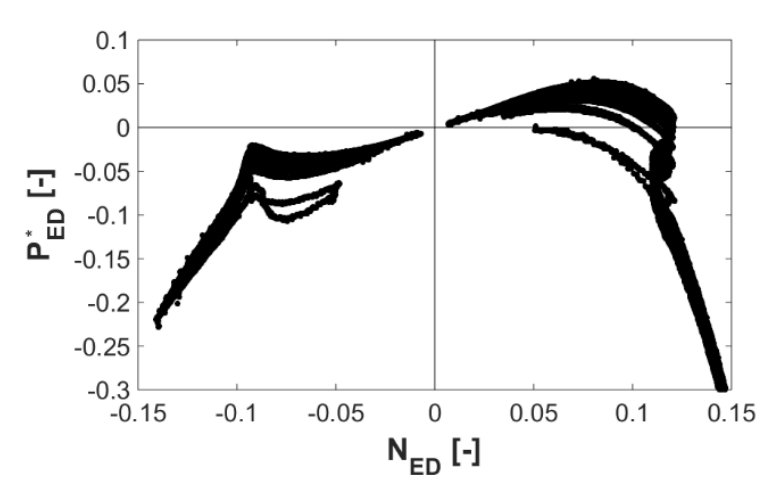

(b)

Figure 10. Resulting 4-quadrant characteristic curves of the PAT obtained by the dynamic measurements method: (a) speed-discharge factor characteristic curve; (b) speed-power factor characteristic curves.

\subsubsection{Resulting Full 3D Hill-Charts}

The resulting efficiency 3D hill-charts obtained by the classical static point-by-point and by the dynamic measurement methods are provided in Figures 11 and 12. From a qualitative point of view, the results obtained by the dynamic method are in a good agreement with the ones obtained by the static method for both tested cases. Indeed, a satisfactory match between the two resulting 3D hill-surfaces (see the superposition of the fitted surfaces in Figure 13) validates the alternative proposed dynamic measurements method. The differences $\varepsilon_{S-D}$ between the two hill-chart surfaces, computed with Equation (10), are mainly due to the quality of the interpolation and due to the number and the position of the available operating points. A more refined grid of operating points for classical static point-by-point method along with a more adapted surface interpolation method should reduce these artificial differences. 
Finally, in the case of the microturbine, if for the static method the measured operating points cross horizontally the final Q-H characteristic, in the case of the dynamic method the intersection with a typical characteristic of a pump is noticed from the distribution of operating points.

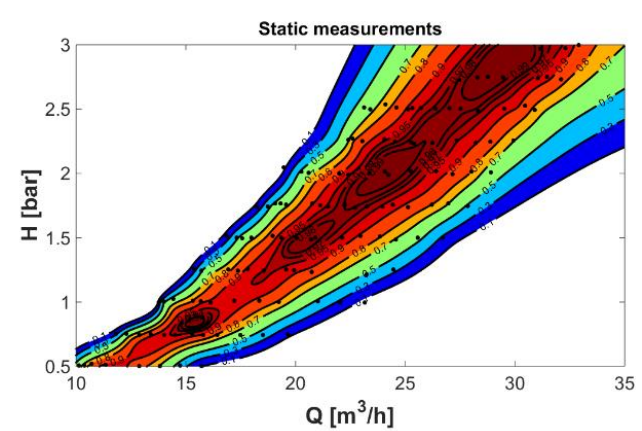

(a)

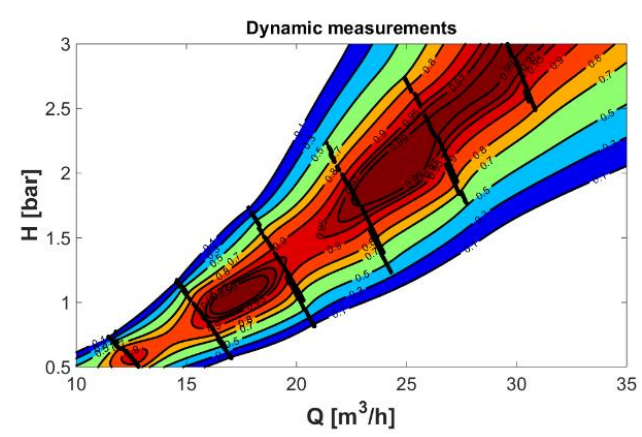

(c)

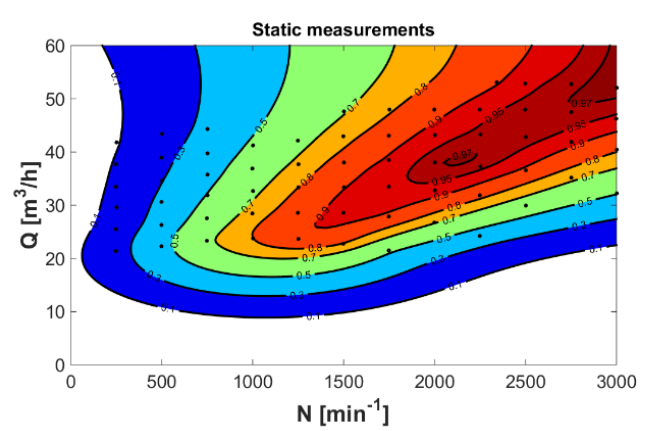

(b)

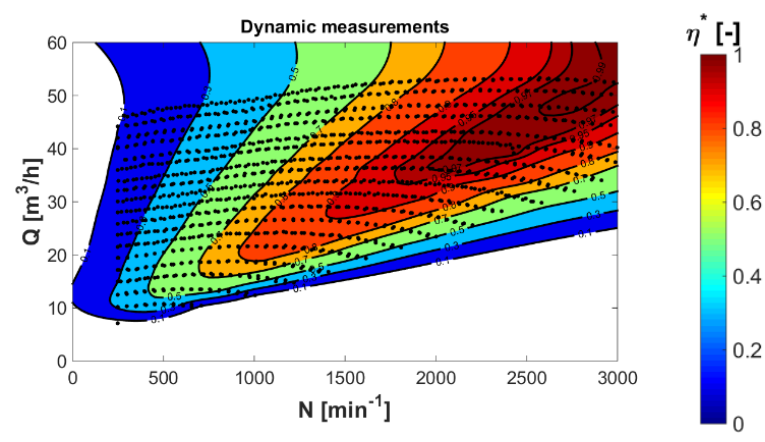

(d)

(e)

Figure 11. Resulting efficiency hill-chart contours obtained by classical static point-by-point and by the dynamic measurement methods: (a) counter-rotating microturbine-result of static measurements; (b) PAT-result of static measurements; (c) counter-rotating microturbine-result of dynamic measurements; (d) PAT—result of dynamic measurements; (e) contour plot scale.

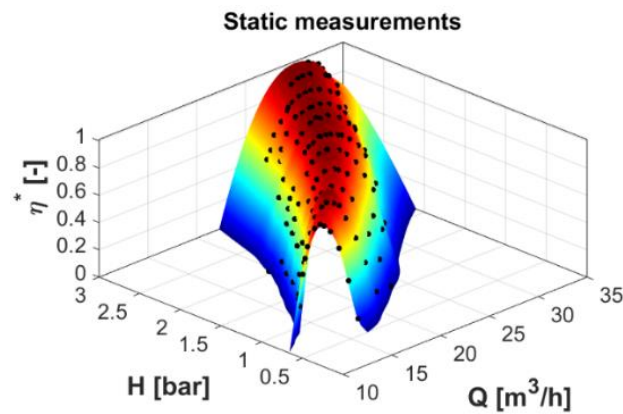

(a)

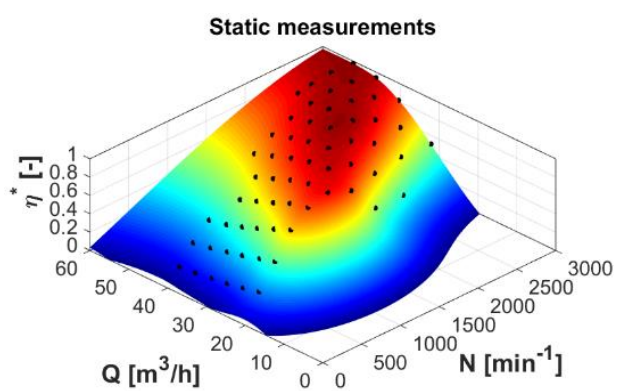

(b)

Figure 12. Cont. 


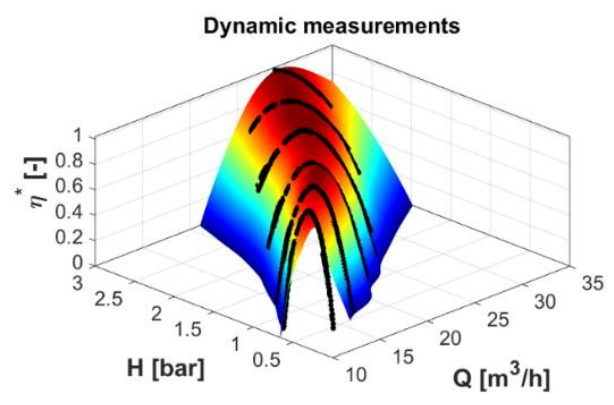

(c)

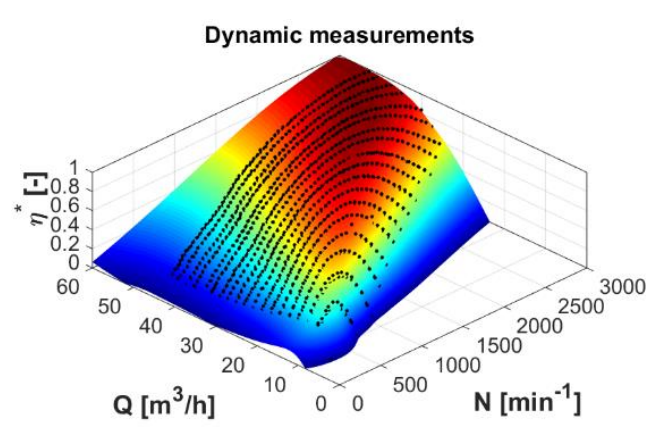

(d)

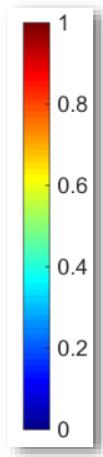

(e)

Figure 12. Resulting efficiency 3D hill-charts obtained by classical static point-by-point and by the dynamic measurement methods: (a) counter-rotating microturbine-result of static measurements; (b) PAT-result of static measurements; (c) counter-rotating microturbine-result of dynamic measurements; (d) PAT—result of dynamic measurements; (e) colour plot scale.

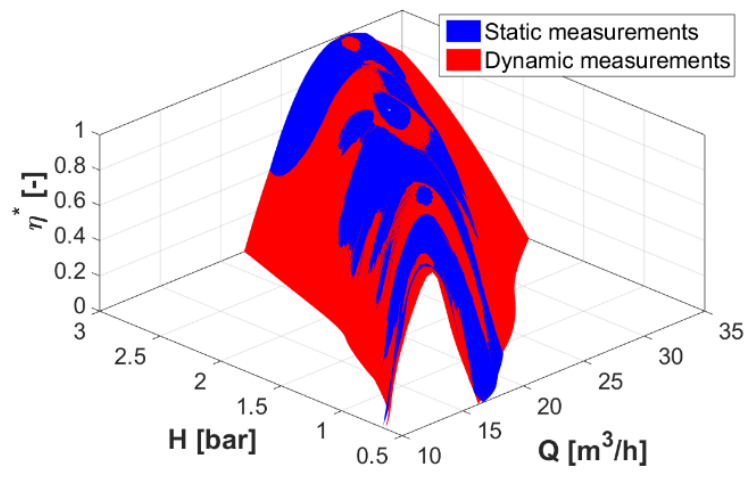

(a)

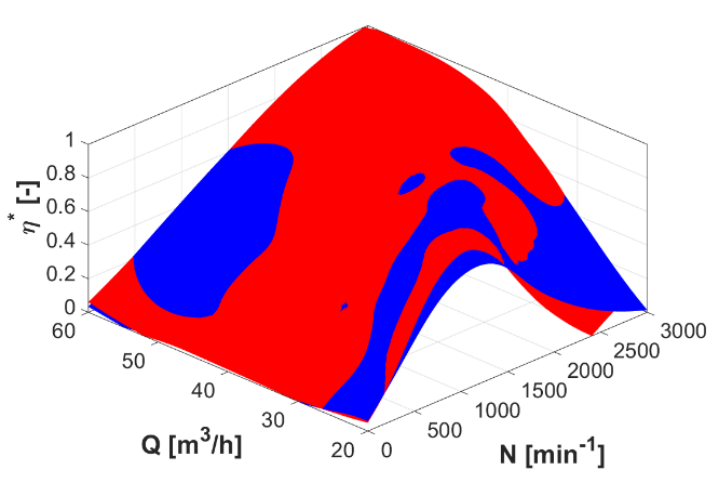

(b)

Figure 13. Validation of resulting 3D hill-charts obtained by the dynamic measurements method with the results of the classical static point-by-point method: (a) comparison for the counter-rotating microturbine; (b) comparison for the PAT.

\section{Discussion}

A final balance between the two investigated testing methods is summarized in Table 7 . The most remarkable difference between the two methods is the total time necessary to retrieve the final 3D hill-chart of a double-regulated turbine. In terms of efficiency difference, the results presented in Figure 14 remains qualitative since they are highly dependent on the shape of the interpolated surfaces using more or less enough information for an irrefutable definition. However, one may state that the differences are general below $\pm 1 \%$ (excepting the regions without enough measurement information to correctly describe the hill-surface), considered acceptable taking into account the amount of information acquired as well as the saved time.

Focusing on the microturbine, the static measurements have been performed for approximately 1000 operating points. Forty man-work hours (one full man-work week) were necessary to calibrate/check the instruments, set the test rig and perform the measurements. Indeed, the classical static point-by-point method requires a considerable time for a complete measurement campaign of the full 3D hill-chart because a consistent number of operating points are necessary. For each operating point, once the operating parameters set, a waiting period of several tens of seconds is systematically required to stabilize the operating point. In the case of dynamic measurements, the total time to calibrate/check the instruments, set the test rig and to perform the measurements is reduced to 
approximately eight man-work hours (one man-work day). The main advantage is the shortest time necessary to obtain the full 3D hill-chart.

$$
\varepsilon_{S-D}=\eta_{\text {Static }}^{*}-\eta_{\text {Dynamic }}^{*}[-]
$$

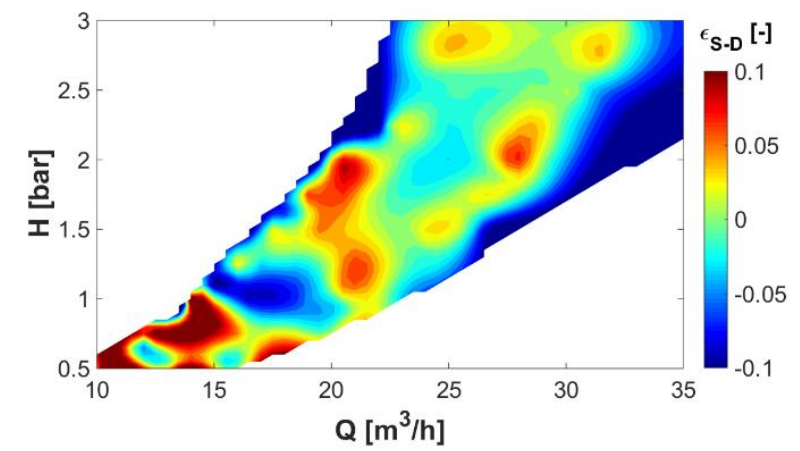

(a)

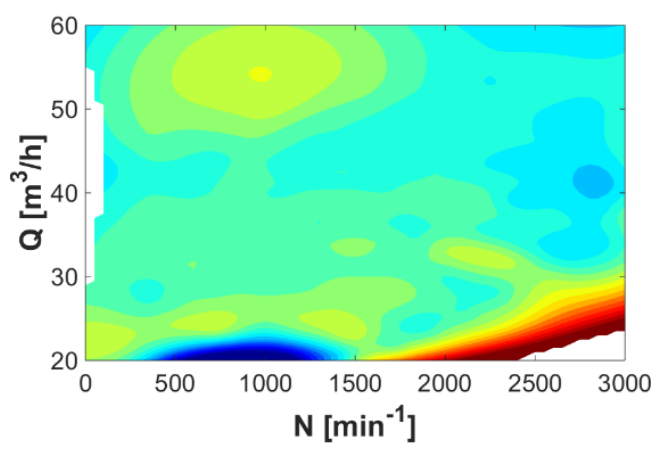

(b)

Figure 14. Resulting contours of difference between the 3D hill-charts obtained by the dynamic measurements method compared with the results of the classical static point-by-point method: (a) result for the counter-rotating microturbine; (b) result in the case of the PAT.

In the case of the PAT (a single regulated machine), the static and the dynamic measurements required more or less the same investigation total time. Anyway, the advantage on the "sliding-gate" method comes from the fact that the method allowed building a more precise 3D hill-chart since the number of acquired measurement points is several thousand times larger than in the case of the static method. Moreover, the dynamic method allowed measuring the S-shaped characteristic curves, which for the classical method can be done only with a special control of the test rig (see the method presented in Hasmatuchi [19]) and requests a considerable time.

Table 7. Final balance between the two testing methods.

\begin{tabular}{ccccc}
\hline \multirow{2}{*}{ Criterion } & \multicolumn{2}{c}{ Microturbine } & \multicolumn{2}{c}{ PAT } \\
\cline { 2 - 5 } & $\begin{array}{c}\text { Static } \\
\text { Measurements }\end{array}$ & $\begin{array}{c}\text { Dynamic } \\
\text { Measurements }\end{array}$ & $\begin{array}{c}\text { Static } \\
\text { Measurements }\end{array}$ & $\begin{array}{c}\text { Dynamic } \\
\text { Measurements }\end{array}$ \\
\hline Testing variable & $11 \times$ testing heads & $9 \times$ pumps' speeds $^{\prime}$ & $10 \times$ pumps' speeds & $19 \times$ pumps' speeds $^{\prime}$ \\
\hline $\begin{array}{c}\text { Turbine runner(s) } \\
\text { speed }\end{array}$ & $\begin{array}{c}13 \times \text { speed ratios, } \\
\text { speeds, } 0 \div 100 \% \\
\text { speed range }\end{array}$ & $\begin{array}{c}11 \times \text { speed ratios, } \\
0 \div 100 \% \text { forth and } \\
\text { back runners speed } \\
\text { variation }\end{array}$ & $\begin{array}{c}12 \times \text { runner speeds, } \\
8.33 \div 100 \% \text { speed } \\
\text { range }\end{array}$ & $\begin{array}{c}0 \div 100 \% \text { forth and } \\
\text { back runner speed } \\
\text { variation }\end{array}$ \\
\hline $\begin{array}{c}\text { Total measured } \\
\text { points/curves }\end{array}$ & $\begin{array}{c}>1000 \text { static } \\
\text { operating points }\end{array}$ & $\begin{array}{c}\sim 70 \text { dynamic } \\
\text { curves }\end{array}$ & $\begin{array}{c}\sim 100 \text { static operating } \\
\text { points }\end{array}$ & 19 dynamic curves \\
\hline Total time & $\sim \mathbf{1}$ men-week & $\sim \mathbf{1}$ men-day & $\sim \mathbf{1 / 2}$ men-day & $\sim \mathbf{1 / 2}$ men-day \\
\hline
\end{tabular}

The dynamic measurements allow for fast detection of eventual hydraulic instabilities in the machine from local hill-chart humps or using fast sensors mounted in key locations (e.g., fast wall pressure sensors, accelerometers, etc.). Today, hydraulic turbines are often operated at operating regimes far from the best efficiency point. The dynamic method allows investigating the machine behaviour and identifying the eventual hydraulic instabilities that can occur on the full operating range. Further, complementary extensive experimental investigations can be performed for the problematic operating regions dominated by hydraulic instabilities.

To cite an eventual drawback of the method, a second digitizer and a relatively higher acquisition frequency are necessary to acquire synchronized dynamic signals from the sensors. The authors 
would recommend keeping the acquisition system dedicated for static measurement on in order to validate the first dynamic measurement curves as well as to calibrate the acceleration/deceleration ramps speeds.

To complete, the same dynamic method has been successfully employed to characterize the efficiency of the coupling between the generator and the gearbox of a hydrokinetic turbine prototype (see Münch-Alligné et al. [34]). Indeed, a manual brake has been used to vary the torque during the tests. Despite the fact that the same load has been applied on the brake lever, it was quasi-impossible to perform repetitive static measurements mainly due to the uncontrollable continuously variable temperature of the brake. The speed-torque-efficiency characteristic of the tested assembly has been successfully constructed from curves at constant brake load while varying the rotational speed of the system (measured dynamically). The full 3D hill-chart has been crossed on different paths during an increase-decrease speed cycle. Finally, the method could be applied for measurements of characteristics not only on hydraulic machines, but also on any other domain (characterization of electrical motors, etc.) where a large number of static operating points is required.

\section{Conclusions}

The implementation of the so-called "sliding-gate" dynamic method to measure in a faster way (up to ten times) the efficiency characteristics on hydraulic turbomachinery has been presented. The alternative testing measurement method has been successfully applied on two different cases dedicated to recover the energy dissipated by release valves of water supply networks: a $2.65 \mathrm{~kW}$ double-regulated laboratory prototype of an in-line axial microturbine with two independent variable speed counter-rotating runners and a $11 \mathrm{~kW}$ multi-stage centrifugal PAT with variable speed.

The measurements have been carried out on the universal test rig of the HES-SO Valais / / Wallis, Switzerland, dedicated to assess hydraulic performances of small-power turbomachines following the IEC standard recommendations. The applied procedure consisted, in a first step, on measuring the 3D hill-chart of a given testing model (turbine or pump) by the classical static point-by-point method. Then, a second digitizer has been installed to acquire synchronized dynamic signals of the sensors in parallel with the existing acquisition/control systems of the test rig and of the testing model. The dynamic measurements of efficiency have been performed at different constant speeds of the test rig recirculating pumps while increasing and/or decreasing the speed of the testing model runner(s) from zero to maximum, and vice versa, slowly enough in order to maintain a steady-state operation. Finally, the resulting 3D efficiency hill-charts of the two tested machines obtained by the dynamic have been compared and validated with the results obtained by the classical static point-by-point measurement method. Moreover, the S-shaped 4-quadrants characteristics of the PAT have been also successfully measured using the dynamic method.

In conclusion, this approach is particularly gainful for small-hydro due to the reduced time required to perform the hydraulic efficiency tests. The same method could be interesting to rapidly identify the regions dominated by hydrodynamic instabilities within the operating range of a turbomachine.

Author Contributions: Wrote the paper, V.H., A.I.B. and C.M.-A.; Conceived and designed the experiments, V.H. and A.I.B.; Performed the experiments and analysed the data, V.H., A.I.B. and S.L.

Funding: This research was funded by [the Swiss National Science Foundation] that supported financially Alin Bosioc through the International Short Visit program [grant number: IZK0Z2_163500, 2015].

Acknowledgments: The present work was also founded by the Swiss Competence Center for Energy Research-Supply of Electricity (SCCER-SoE), granted by the Swiss Commission for Technology and Innovation (CTI). The development of the microturbine was carried out in the framework of Hydro VS project, in partnership with the Laboratory for Hydraulic Machines from École Polytechnique Fédérale de Lausanne, Switzerland, granted by the program The Ark Energy of the Ark - the foundation for innovation in Valais, Switzerland. Finally, the PAT performance measurements have been done in the framework of the Savièse project, financially supported by Cimark and by the program The Ark Energy of the Ark, Valais, Switzerland. ClaVal Switzerland is also thanked for all their support with the release valves supply. 
Conflicts of Interest: The authors declare no conflict of interest.

\section{Nomenclature}

\begin{tabular}{|c|c|c|}
\hline \multicolumn{3}{|c|}{ Symbols } \\
\hline \multicolumn{3}{|r|}{ Runner outlet diameter } \\
\hline $\mathrm{E}$ & {$\left[\mathrm{J} \cdot \mathrm{kg}^{-1}\right]$} & Specific energy \\
\hline$g$ & {$\left[\mathrm{~m} \cdot \mathrm{s}^{-2}\right]$} & Gravity \\
\hline $\mathrm{H}$ & {$[\mathrm{m}]$} & Head \\
\hline $\mathrm{N}$ & {$\left[\min ^{-1}\right]$} & Runner rotational speed \\
\hline $\mathrm{N}_{\mathrm{ED}}$ & {$[-]$} & Speed factor \\
\hline $\mathrm{P}$ & {$[W]$} & Power \\
\hline $\mathrm{P}^{*} \mathrm{ED}$ & {$[-]$} & $\begin{array}{l}\text { Power factor (based on } \\
\text { mechanical/electrical power) }\end{array}$ \\
\hline Q & {$\left[\mathrm{m}^{3} \cdot \mathrm{s}^{-1}\right]$} & Discharge \\
\hline$Q_{E D}$ & {$[-]$} & Discharge factor \\
\hline $\mathrm{T}_{\mathrm{mec}}$ & {$[\mathrm{N} \cdot \mathrm{m}]$} & Runner mechanical torque \\
\hline$\eta$ & {$[\%]$} & Efficiency \\
\hline$\eta^{*}$ & {$[-]$} & Dimensionless efficiency \\
\hline$\eta^{* \prime}$ & {$[-]$} & $\begin{array}{l}\text { Dimensionless efficiency } \\
\text { fluctuation }\end{array}$ \\
\hline$\eta^{*}$ fit & {$[-]$} & Fitted dimensionless efficiency \\
\hline$\eta^{* \prime}$ STD & {$[-]$} & $\begin{array}{l}\text { Efficiency fluctuation Standard } \\
\text { Deviation }\end{array}$ \\
\hline$\rho$ & {$\left[\mathrm{kg} \cdot \mathrm{m}^{-3}\right]$} & Water density \\
\hline$\omega$ & {$\left[\mathrm{rad} \cdot \mathrm{s}^{-1}\right]$} & Runner angular speed \\
\hline \multicolumn{3}{|c|}{ Subscripts } \\
\hline $\mathrm{A}, \mathrm{B}$ & \multicolumn{2}{|c|}{ 1st, 2 nd independent runners } \\
\hline e & \multicolumn{2}{|c|}{ Energetic losses } \\
\hline elec & \multicolumn{2}{|l|}{ Electrical } \\
\hline $\mathrm{h}$ & \multicolumn{2}{|c|}{ Hydraulic } \\
\hline $\mathrm{m}$ & \multicolumn{2}{|c|}{ Bearing losses } \\
\hline mec & \multicolumn{2}{|c|}{ Mechanical } \\
\hline $\mathrm{q}$ & \multicolumn{2}{|c|}{ Volumetric losses } \\
\hline $\mathrm{rm}$ & \multicolumn{2}{|c|}{ Disc friction losses } \\
\hline
\end{tabular}

\section{References}

1. World Energy Council. World Energy Resources Hydropower. 2016. Available online: https:// www.worldenergy.org/wp-content/uploads/2017/03/WEResources_Hydropower_2016.pdf (accessed on 20 July 2018).

2. Official Website of the Swiss Federal Office for Energy. Available online: http://www.bfe.admin.ch/themen/ 00526/00541/00543/index.html?lang=fr\&dossier_id=00772 (accessed on 20 July 2018).

3. Official Website of the Swiss Federal Office for Energy. Available online: http:/ /www.bfe.admin.ch/themen/ 00490/00491/00492/index.html?lang=fr (accessed on 20 July 2018).

4. Ruprecht, A.; Bauer, C.; Gentner, C.; Lein, G. Parallel Computation of Stator-Rotor Interaction in an Axial Turbine. In Proceedings of the ASME PVP Conference, CFD Symposium, Boston, MA, USA, 1-5 August 1999; pp. 1-9.

5. Ruprecht, A.; Bauer, C.; Heitele, M. Unsteady Forces on the Blading of an Axial Turbine Caused by Stator-Rotor Interaction. In Proceedings of the IAHR WG “The Behaviour of Hydraulic Machinery under Steady Oscillatory Conditions", Brno, Czech Republic, 7-9 September 1999.

6. Alexander, K.V.; Giddens, E.P.; Fuller, A.M. Axial-Flow Turbines for Low Head Microhydro Systems. Renew. Energy 2009, 34, 35-47. [CrossRef] 
7. Samora, I.; Hasmatuchi, V.; Münch-Alligné, C.; Franca, M.J.; Schleiss, A.J.; Ramosa, H.M. Experimental characterization of a five blade tubular propeller turbine for pipe inline installation. Renew. Energy 2016, 95, 356-366. [CrossRef]

8. Sonohata, R.; Fukutomi, J.; Shigemitsu, T. Study on Contra-Rotating Small-Sized Axial Flow Hydro Turbine. Open J. Fluid Dyn. 2012, 2, 318-323. [CrossRef]

9. Münch-Alligné, C.; Richard, S.; Meier, B.; Hasmatuchi, V.; Avellan, F. Numerical Simulations of a Counter Rotating Micro Turbine. In Advances in Hydroinformatics. SIMHYDRO 2012-New Frontiers of Simulation; Gourbesville, P., Cunge, J., Caignaert, G., Eds.; Springer: Singapore, 2014; pp. 363-373, ISBN 978-981-4451-41-3.

10. Biner, D.; Hasmatuchi, V.; Violante, D.; Richard, S.; Chevailler, S.; Andolfatto, L.; Avellan, F.; Münch, C. Engineering \& Performance of DuoTurbo: Microturbine with Counter-Rotating Runners. Earth Environ. Sci. 2016, 49, 102013. [CrossRef]

11. Vagnoni, E.; Andolfatto, L.; Richard, S.; Münch-Alligné, C.; Avellan, F. Hydraulic performance evaluation of a micro-turbine with counter rotating runners by experimental investigation and numerical simulation. Renew. Energy 2018, 126, 943-953. [CrossRef]

12. Hasmatuchi, V.; Botero, F.; Gabathuler, S.; Münch, C. Design and Control of a New Hydraulic Test Rig for Small Hydro Turbines. Int. J. Hydropower Dams 2015, 22, 51-60.

13. Münch-Alligné, C.; Avellan, F. Exploitation du potentiel de la petite hydraulique: Situation actuelle et exemple de développement. Bull. SEV 2013, 2, 41-45.

14. Almquist, C.W.; March, P.A.; Franseen, H.W. The Sliding Gate Method: A Better Way of Turbine Efficiency Testing? Hydro Rev. 1997, XVI, 44-53.

15. Almquist, C.W.; Bickford, B. Further Development of the Sliding Gate Method for Hydroturbine Efficiency Testing. Water Power 2005, XIV, 1-14.

16. Abgottspon, A.; Staubli, T. Index Tests of a Francis Unit Using the Sliding Gate Method. In Proceedings of the International Conference on Hydraulic Efficiency Measurements, Milano, Italy, 3-6 September 2008.

17. Abgottspon, A.; Staubli, T.; Felix, D.; Albayrak, I.; Boes, R.M. Monitoring Suspended Sediment and Turbine Effciency. Hydro Rev Worldwide, 1 July 2014; p. H1.

18. Casanova, F. Failure Analysis of the Draft Tube Connecting Bolts of a Francis-Type Hydroelectric Power Plant. Eng. Fail. Anal. 2009, 16, 2203-2208. [CrossRef]

19. Hasmatuchi, V. Hydrodynamics of a Pump-Turbine Operating at Off-Design Conditions in Generating Mode. Ph.D. Thesis, École Polytechnique Fédérale de Lausanne, Lausanne, Switzerland, 2012.

20. Jacob, T.; Prenat, J.E. Francis Turbine Surge: Discussion and Data Base. In Hydraulic Machinery and Cavitation; Cabrera, E., Espert, V., Martinez, F., Eds.; Springer: Dordrecht, The Netherlands, 1996; pp. 855-864, ISBN 978-94-010-9387-3.

21. International Electrotechnical Committee. Hydraulic Turbines, Storage Pumps and Pump-Turbines-Model Acceptance Tests of International Standard IEC 60193, 2nd ed.; IEC: Geneva, Switzerland, 1999.

22. Biner, D.; Hasmatuchi, V.; Avellan, F.; Münch-Alligné, C. Design \& Performance of a Hydraulic Micro-Turbine with Counter-Rotating Runners. In Proceedings of the 5th International Youth Conference on Energy, Pisa, Italy, 27-30 May 2015; pp. 1-10. [CrossRef]

23. Melly, D.; Horta, R.; Münch, C.; Biner, H.; Chevailler, S. Development of a PM-Generator for a Counter-Rotating Micro-Hydro Turbine. In Proceedings of the XXI International Conference on Electrical Machines, Berlin, Germany, 2-5 September 2014; pp. 124-129.

24. Orchard, B.; Klos, S. Pumps as Turbines for Water Industry. World Pumps 2009, 8, 22-23. [CrossRef]

25. Williams, A.A. Pumps as Turbines for Low Cost Micro-Hydro Power. Renew. Energy 1996, 9, $1227-1234$. [CrossRef]

26. Ramos, H.; Borga, A. Pumps as Turbines: An Unconventional Solution to Energy Production. Urban Water 1999, 1, 261-263. [CrossRef]

27. Carravetta, A.; Derakhshan, H.S.; Ramos, H.M. Pumps as Turbines-Fundamentals and Applications, 1st ed.; Springer: Berlin, Germany, 2018.

28. Motwani, K.H.; Jain, S.V.; Patel, R.N. Cost Analysis of Pump as Turbine for Pico Hydropower Plants-A Case Study. Procedia Eng. 2013, 51, 721-726. [CrossRef]

29. Garay, P. Using Pumps as Hydroturbines. Hydro Rev. 1990, 9, 52-61. 
30. Chapallaz, J.M.; Eichenberger, P.; Fischer, G. Manual on Pumps Used as Turbines; Vieweg \& Sohn Verlagsgesellschaft mbH: Braunschweig, Germany, 1992.

31. Derakhshan, S.; Nourbakhsh, A. Experimental Study of Characteristic Curves of Centrifugal Pumps Working as Turbines in Different Specific Speeds. Exp. Therm. Fluid Sci. 2008, 32, 800-807. [CrossRef]

32. Hasmatuchi, V.; Bosioc, A.; Münch-Alligné, C. On the Dynamic Measurements of Hydraulic Characteristics. Earth Environ. Sci. 2016, 49, 062001. [CrossRef]

33. Hasmatuchi, V.; Bosioc, A.; Luisier, S.; Münch-Alligné, C. Dynamic Efficiency Measurements on Hydraulic Turbomachinery: Examples of Implementation and Validation. In Proceedings of the 11th International Conference on Hydraulic Efficiency Measurement, Linz, Austria, 24-26 August 2016.

34. Münch-Alligné, C.; Schmid, J.; Richard, S.; Gaspoz, A.; Brunner, N.; Hasmatuchi, V. Experimental Assessment of a New Kinetic Turbine Performance for Artificial Channels. Water 2018, 10, 311. [CrossRef]

(C) 2018 by the authors. Licensee MDPI, Basel, Switzerland. This article is an open access article distributed under the terms and conditions of the Creative Commons Attribution (CC BY) license (http://creativecommons.org/licenses/by/4.0/). 